\title{
STOCHASTIC VELOCITY MOTIONS AND PROCESSES WITH RANDOM TIME
}

\author{
ALESSANDRO DE GREGORIO, ${ }^{*}$ Sapienza University of Rome
}

\begin{abstract}
The aim of this paper is to analyze a class of random processes which models the motion of a particle on the real line with random velocity and subject to the action of friction. The speed randomly changes when a Poissonian event occurs. We study the characteristic and moment generating functions of the position reached by the particle at time $t>0$. We are able to derive the explicit probability distributions in a few cases. The moments are also widely analyzed. For the random motions having an explicit density law, further interesting probabilistic interpretations emerge if we consider randomly varying time. Essentially, we consider two different types of random time, namely Bessel and gamma times, which contain, as particular cases, some important probability distributions (e.g. Gaussian, exponential). For the random processes built by means of these compositions, we derive the probability distributions for a fixed number of Poisson events. Some remarks on possible extensions to random motions in higher spaces are proposed. We focus our attention on the persistent planar random motion.
\end{abstract}

Keywords: Bessel process; gamma process; iterated Brownian motion; Struve function; Laplace distribution; random flight; random time; telegraph process

2010 Mathematics Subject Classification: Primary 60G07; 26B15

\section{Introduction}

Diffusion processes have a central position in the theory of probability. Nevertheless, their main shortcoming is the unboundeness of the first variation. For this reason, a diffusion process is often not suitable to describe the real motion and many researchers have proposed alternative models having finite speed.

The prototype of random motions with finite velocity is the telegraph process. By assuming that the change of direction is governed by a homogeneous Poisson process $N(t), t>0$, with rate $\lambda>0$, we can define the telegraph process as

$$
T(t)=V(0) \sum_{j=1}^{N(t)+1}\left(s_{j}-s_{j-1}\right)(-1)^{j-1},
$$

where $V(0)$ is the initial velocity assuming the values $+c$ or $-c$ with probability $\frac{1}{2}$ and the times $s_{j}$ are the instants in which the $j$ th Poisson event occurs. Furthermore, $T(t), t>0$, is linked with the hyperbolic partial differential equations, because its density law is the fundamental solution of the equation

$$
\frac{\partial^{2} u}{\partial t^{2}}+2 \lambda \frac{\partial u}{\partial t}=c^{2} \frac{\partial^{2} u}{\partial x^{2}}
$$

Received 11 January 2010; revision received 16 June 2010.

* Postal address: Department of Statistical Science, Sapienza University of Rome, Piazzale Aldo Moro, 5 - 00185, Rome, Italy. Email address: alessandro.degregorio@uniroma1.it 
The telegraph process has been studied by several authors; see, for example, Orsingher (1990), Foong and Kanno (1994), Di Crescenzo (2001), Stadje and Zacks (2004) and Zacks (2004). This model seems to be suitable to describe the real motion and it emerges in different fields. In physical mathematics the connection between the telegraph process and the electromagnetic theory strongly emerges. In particular, (1.2) describes the propagation of a damped wave along a wire. Weiss (2002) provided an interesting review of physical applications of the process $T(t), t>0$. Models governed by hyperbolic differential equations and, in particular, telegraph equations have been exploited to describe the movement of chemotaxis (see Hillen and Stevens (2000)). The telegraph process is useful in ecology to model the displacement of wild animals on the soil (see Holmes et al. (1994)). Indeed, this model preserves the property of animals to move at finite velocity along the same direction. Di Crescenzo and Pellerey (2002) introduced the geometric telegraph process as a model to describe the dynamics of the price of risky assets, i.e. the authors replaced the standard Brownian motion with the standard telegraph process. Ratanov (2007a), (2007b) proposed a model of financial markets using the telegraph process with two different velocities (as the risky asset tends upward or downward) and jumps occurring at switching velocities. Mazza and Rulliere (2004) established a link between hitting times associated with the risk process (time of ruin of the insurance company) and the telegrapher's motion.

A statistical analysis of the random model $T(t), t>0$, has been performed by De Gregorio and Iacus (2008) and Iacus and Yoshida (2009), when the sample path is observed at discrete times.

In this paper we will analyze a one-dimensional random motion which generalizes $T(t)$, $t>0$. At time $t>0$, the random speed of the motion is defined by $v=c \cos \theta$, where $c$ is a positive constant and $\theta$ is a random variable with density given by

$$
f_{v}(\theta)=\frac{\Gamma(v+1)}{\sqrt{\pi} \Gamma(v+1 / 2)} \sin ^{2 v} \theta, \quad \theta \in(0, \pi), v \geq 0 .
$$

So, we consider a particle starting from the origin, choosing initially a velocity $c \cos \theta_{1}$ with probability law given by $f_{v}(\theta)$. The particle travels maintaining its motion with the same velocity until a Poisson event occurs. Now, the particle changes velocity independently to the previous velocity according to $f_{v}(\theta)$ again, and so on. At time $t>0$, we indicate the particle position on the real line with $X_{v}(t)$.

The function $f_{v}(\theta)$ permits us to define a random motion in which the small displacements have a greater probability than the large displacements. Therefore, the random walker moves with friction. Indeed, every object or particle moving on a surface suffers an effect due to the friction of the surface itself. Then, the particle will tend to go away from the starting point slowly. If $v=0$ then $f_{0}(\theta)=1 / \pi$ becomes the uniform distribution on the semicircle with radius 1 . In this last case the particle moves without friction.

We study the conditional characteristic and moment generating functions of $X_{v}(t), t>0$. Fixing the number of Poisson events, we are able to derive the conditional probability distribution in the cases $v=0$ and $v=1$. Therefore, let $N(t), t>0$, denote the underlying Poisson process governing the changes of the velocity. Then we have

$$
\begin{aligned}
& \frac{\mathrm{P}\left(X_{0}(t) \in \mathrm{d} x \mid N(t)=n\right)}{\mathrm{d} x}=\frac{\Gamma(n / 2) \Gamma(n / 2+1)}{2 \pi \Gamma(n)}\left(\frac{2}{c t}\right)^{n}\left(c^{2} t^{2}-x^{2}\right)^{(n-1) / 2}, \\
& \frac{\mathrm{P}\left(X_{1}(t) \in \mathrm{d} x \mid N(t)=n\right)}{\mathrm{d} x}=\frac{\Gamma(n+1) \Gamma(n+2)}{2 \pi \Gamma(2 n+2)}\left(\frac{2}{c t}\right)^{2 n+2}\left(c^{2} t^{2}-x^{2}\right)^{n+1 / 2},
\end{aligned}
$$


with $n \geq 0$ and $|x|<c t$. These results permit us to point out the relationship between $X_{0}(t), t>0$, and $X_{1}(t), t>0$, and the random flights studied by several authors, such as Stadje (1987), (1989), Kolesnik and Orsingher (2005), De Gregorio and Orsingher (2006), Kolesnik (2006), and Orsingher and De Gregorio (2007). A random flight is a continuoustime random walk defined similarly to $X_{v}(t)$, but with its direction chosen uniformly on an hypersphere. By means of the above probabilities we can claim that, in distribution, $X_{0}(t)$ and $X_{1}(t)$ respectively correspond to the projection onto the real axis of a planar random flight and a four-dimensional random flight.

Furthermore, we derive the first two moments of $X_{v}(t), t>0$, while, for $v=0$, we are able to make explicit the moments of order $p$ by means of special functions.

In the second part of this paper, we focus our attention on the random motions $X_{0}(t), t>0$, and $X_{1}(t), t>0$, evolving up to a random time, leading to interesting interpretations of the related conditional probability distributions. In other words, we will introduce families of random times, containing as particular cases some important random variables. In the probabilistic literature there are several papers devoted to the analysis of the properties of stochastic processes with random times. For example, the Brownian motion with Brownian time (iterated Brownian motion) has been studied in Burdzy (1993), Khoshnevisan and Lewis (1996), Allouba (2002), DeBlassie (2004), Nane (2006), and Orsingher and Beghin (2009). The iterated Brownian motion has been proposed as a model for a diffusion in a crack (see Burdzy and Khoshnevisan (1998)). Beghin and Orsingher (2009) studied a planar random motion with Brownian times; the authors provided the conditional probability on the number of the events of a fractional Poisson process.

In this work, as random time, we consider the well-known Bessel process

$$
B_{d}(t)=\sqrt{\sum_{i=1}^{d} W_{i}^{2}(t)}, \quad t>0 \text { and } d \geq 1,
$$

where the $W_{i}(t) \mathrm{s}$ are independent Brownian motions. Under the condition that $v_{m}>d / 2-1$, $m=0,1$, the following result holds:

$$
\begin{aligned}
& \mathrm{P}\left\{X_{m}\left(B_{d}(t)\right) \in \mathrm{d} x \mid N\left(B_{d}(t)\right)=n\right\} \\
& \quad=\frac{\mathrm{d} x}{B\left(d / 2, v_{m}-d / 2+1\right)} \int_{0}^{1} w^{d / 2-1}(1-w)^{v_{m}-d / 2} \frac{\mathrm{e}^{-x^{2} / 2 c^{2} t w}}{\sqrt{2 \pi t w} c} \mathrm{~d} w,
\end{aligned}
$$

where $v_{0}=n / 2, v_{1}=n+1, n \geq 1$, and $B(a, b)$ is a beta function with $a>0$ and $b>0$. Hence, the random motion $X_{m}$ stopped at a Bessel random time drastically changes its probability distribution, which becomes Gaussian with variance given by a beta random variable (up to the scale factor $c^{2} t$ ).

For $d=1$, the Bessel process becomes a reflected (around 0) Brownian motion $|W(t)|$, that is, the Brownian time arising in the iterated Brownian motion, while, for $d=2$, we obtain a random clock distributed as a Rayleigh random variable. We provide the unconditional densities of $X_{m}\left(B_{d}(t)\right), t>0$, in these two cases.

Other relationships will be pointed out when considering the composition with the sojourn time of a Brownian motion on the positive axis.

Let $G_{\alpha}(t), t>0$, be a gamma process with $\alpha>0$, that is, a random process with probability law given by $g_{\alpha}(s, t)=t^{\alpha} s^{\alpha-1} \mathrm{e}^{-t s} / \Gamma(\alpha), s>0, t>0$. The gamma random variable arises 
in various applications and it is useful to model the lifetime of a phenomenon. For this reason, we deal with a gamma time and, for $X_{m}\left(G_{\alpha}(t)\right), t>0$, obtain

$$
\begin{aligned}
\mathrm{P}\left\{X_{m}\left(G_{\alpha}(t)\right) \in \mathrm{d} x \mid N\left(G_{\alpha}(t)\right)=n\right\} \\
=\frac{\mathrm{d} x}{\Gamma((\alpha+1) / 2) B\left(\alpha / 2, v_{m}-\alpha / 2+1\right)} \\
\quad \times \int_{0}^{1} \mathrm{~d} w w^{\alpha / 2-1}(1-w)^{v_{m}-\alpha / 2} \frac{t}{\sqrt{\pi w} c}\left(\frac{t|x|}{2 c \sqrt{w}}\right)^{\alpha / 2} K_{-\alpha / 2}\left(\frac{t|x|}{c \sqrt{w}}\right)
\end{aligned}
$$

with $v_{m}>\alpha / 2-1$ and $n \geq 1$, where $K_{\mu}(x)=(1 / 2)(x / 2)^{\mu} \int_{0}^{\infty} \mathrm{e}^{-z-x^{2} / 4 z} z^{-\mu-1} \mathrm{~d} z$.

To complete the discussion on random times, we deal with a random clock obtained by mixing $B_{d}(t)$ and $G_{\alpha}(t)$, and study the effect of the random motion $X_{m}(t)$ on the probabilistic structure.

It is not a difficult task to extend the previous results to the planar and four-dimensional random flights with randomly varying time. Moreover, in Section 4, we will discuss the possibility of a planar random flight with drift, which is persistent along a portion of the surface.

\section{Moving randomly with friction}

Let us consider a random motion which describes the displacements of a particle starting from the origin of the real axis. The particle moves forward or backward with random velocity $v=c \cos \theta$, where $c$ is a positive constant and $\theta$ is a random variable having density

$$
f_{v}(\theta)=\frac{\Gamma(v+1)}{\sqrt{\pi} \Gamma(v+1 / 2)} \sin ^{2 v} \theta,
$$

with $\theta \in(0, \pi)$ and $v \geq 0$. Therefore, the particle moves with a velocity, randomly chosen on the $x$-component of the unit semicircle according to (2.1), and continues to move with this velocity until a Poisson event occurs and another velocity is chosen independently from the previous velocity. The position at time $t$ of the particle is defined as

$$
X_{\nu}(t)=c \sum_{j=1}^{N(t)+1}\left(s_{j}-s_{j-1}\right) \cos \theta_{j}
$$

where $N(t), t>0$, represents the underlying homogeneous Poisson process with rate $\lambda>0$ governing the changes of velocity, the $s_{j}, j=1, \ldots, N(t)+1\left(s_{0}=0, s_{N(t)+1}=t\right)$, are the times of occurrence of the $j$ th Poisson event, and the $\theta_{j} \mathrm{~s}$ are independent random variables distributed as in (2.1). Furthermore, $N(t)$ and $\theta_{j}$ are independent. From (2.2), it emerges that $X_{v}(t), t>0$, is a telegraph-type process similar to $T(t)$ defined in (1.1). We note that $X_{v}(t)$ has an infinite number of possible velocities and it has no necessarily alternating directions. Furthermore, the particle at time $t$ is located inside the interval $(-c t, c t)$, and $X_{v}(t)$ has a fully absolutely continuous probability distribution, whilst in the law of $T(t)$ a singular component appears (see Orsingher (1990)).

We underline that, for values of $\theta$ close to $\pi / 2$, the density law $f_{\nu}(\theta)$ assigns a probability mass greater than 1 near 0 or $\pi$. This means that the process slowly moves away from the starting point. This represents the effect of the friction of the surface on which the particle performs its motion. When $v$ assumes high values, the density $f_{v}(\theta)$ is highly concentrated 


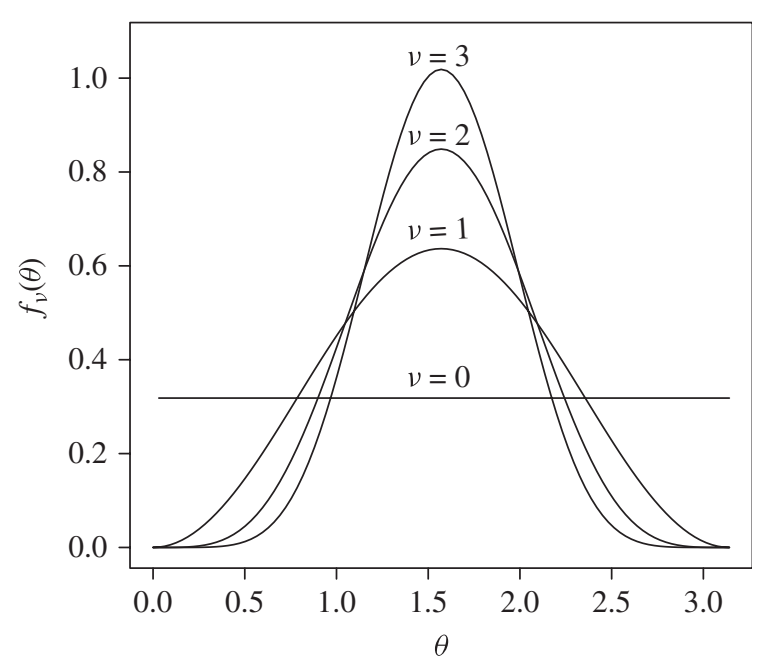

Figure 1: The behavior of $f_{v}(\theta)$ for $v=0,1,2,3$.

around $\pi / 2$ (see Figure 1) and then the motion is slowed down. For this reason, $v$ represents the level of friction to which the motion is subject. In other words, $X_{v}(t), t>0$, defines a whole class of random motions indexed by the parameter $v$, namely the level of friction. For $v=0$, we obtain again the uniform distribution on the semicircle with radius 1 and $X_{0}(t)$ is exactly the $x$-component of a planar random flight studied in, for example, Stadje (1987) and Kolesnik and Orsingher (2005).

Our first result concerns the conditional characteristic function of $X_{v}(t), t>0$, for a fixed number of Poisson events during the time interval $[0, t]$.

Theorem 2.1. The conditional characteristic function of $X_{v}(t), t>0$, is equal to

$$
\mathrm{E}\left\{\mathrm{e}^{\mathrm{i} \alpha X_{v}(t)} \mid N(t)=n\right\}=\frac{n !}{t^{n}}\left(2^{v} \Gamma(v+1)\right)^{n+1} \int_{0}^{t} \mathrm{~d} s_{1} \cdots \int_{s_{n-1}}^{t} \mathrm{~d} s_{n} \prod_{j=1}^{n+1} \frac{J_{v}\left(\alpha c\left(s_{j}-s_{j-1}\right)\right)}{\left(\alpha c\left(s_{j}-s_{j-1}\right)\right)^{v}}
$$

with $n \geq 1$, while, for $N(t)=0$, we have

$$
\mathrm{E}\left\{\mathrm{e}^{\mathrm{i} \alpha X_{v}(t)} \mid N(t)=0\right\}=\left(\frac{2}{\alpha c t}\right)^{v} \Gamma(v+1) J_{v}(\alpha c t),
$$

where

$$
J_{v}(x)=\sum_{k=0}^{\infty} \frac{(-1)^{k}(x / 2)^{2 k+v}}{\Gamma(k+1) \Gamma(k+v+1)}
$$

is the well-known Bessel function of first kind, with $x \in \mathbb{R}$ and $v \in \mathbb{R}$.

Proof. In order to prove (2.3) and (2.4), we observe that the Bessel function $J_{v}(x)$ admits the integral representation

$$
J_{v}(x)=\frac{(x / 2)^{v}}{\Gamma(v+1 / 2) \Gamma(1 / 2)} \int_{0}^{\pi} \mathrm{e}^{\mathrm{i} x \cos \phi} \sin ^{2 v} \phi \mathrm{d} \phi
$$


with $\operatorname{Re}(v+1 / 2)>0$. For $n \geq 1$, we obtain

$$
\begin{aligned}
\mathrm{E}\left\{\mathrm{e}^{\mathrm{i} \alpha X_{v}(t)} \mid N(t)=n\right\} & \\
= & \frac{n !}{t^{n}} \int_{0}^{t} \mathrm{~d} s_{1} \cdots \int_{s_{n-1}}^{t} \mathrm{~d} s_{n} \frac{\Gamma(v+1)}{\sqrt{\pi} \Gamma(v+1 / 2)} \int_{0}^{\pi} \sin ^{2 v} \theta_{1} \mathrm{~d} \theta_{1} \cdots \\
& \times \frac{\Gamma(v+1)}{\sqrt{\pi} \Gamma(v+1 / 2)} \int_{0}^{\pi} \sin ^{2 v} \theta_{n+1} \mathrm{~d} \theta_{n+1} \exp \left\{\mathrm{i} \alpha c \sum_{j=1}^{n+1}\left(s_{j}-s_{j-1}\right) \cos \theta_{j}\right\} \\
= & \frac{n !}{t^{n}} \int_{0}^{t} \mathrm{~d} s_{1} \cdots \int_{s_{n-1}}^{t} \mathrm{~d} s_{n} \prod_{j=1}^{n+1}\left\{\frac{\Gamma(v+1)}{\sqrt{\pi} \Gamma(v+1 / 2)} \int_{0}^{\pi} \mathrm{e}^{\mathrm{i} \alpha c\left(s_{j}-s_{j-1}\right) \cos \theta_{j}} \sin ^{2 v} \theta_{j} \mathrm{~d} \theta_{j}\right\} \\
= & \frac{n !}{t^{n}}\left(2^{v} \Gamma(v+1)\right)^{n+1} \int_{0}^{t} \mathrm{~d} s_{1} \cdots \int_{s_{n-1}}^{t} \mathrm{~d} s_{n} \prod_{j=1}^{n+1} \frac{J_{v}\left(\alpha c\left(s_{j}-s_{j-1}\right)\right)}{\left(\alpha c\left(s_{j}-s_{j-1}\right)\right)^{v}},
\end{aligned}
$$

where in the last step we used the integral representation (2.5). For $N(t)=0$, the position of the particle at time $t$ is $X(t)=c t \cos \theta$. Then

$$
\begin{aligned}
\mathrm{E}\left\{\mathrm{e}^{\mathrm{i} \alpha X_{v}(t)} \mid N(t)=0\right\} & =\frac{\Gamma(v+1)}{\sqrt{\pi} \Gamma(v+1 / 2)} \int_{0}^{\pi} \mathrm{e}^{\mathrm{i} \alpha c t \cos \theta} \sin ^{2 v} \theta \mathrm{d} \theta \\
& =\left(\frac{2}{\alpha c t}\right)^{v} \Gamma(v+1) J_{v}(\alpha c t) .
\end{aligned}
$$

This completes the proof.

It is interesting to observe that (2.3) has the same structure as the characteristic function derived in the problem of $d$-dimensional random flights (see Equation (2.3) of Orsingher and De Gregorio (2007)), where the parameter $v$ is replaced by $d / 2-1$.

For the moment generating function, we present the following theorem.

Theorem 2.2. The conditional moment generating function of $X_{v}(t), t>0$, becomes

$$
\mathrm{E}\left\{\mathrm{e}^{\beta X_{v}(t)} \mid N(t)=n\right\}=\frac{n !}{t^{n}}\left(2^{v} \Gamma(v+1)\right)^{n+1} \int_{0}^{t} \mathrm{~d} s_{1} \cdots \int_{s_{n-1}}^{t} \mathrm{~d} s_{n} \prod_{j=1}^{n+1} \frac{I_{v}\left(\beta c\left(s_{j}-s_{j-1}\right)\right)}{\left(\beta c\left(s_{j}-s_{j-1}\right)\right)^{v}}
$$

with $n \geq 1$, while, if $N(t)=0$, we have

$$
\mathrm{E}\left\{\mathrm{e}^{\beta X_{v}(t)} \mid N(t)=0\right\}=\left(\frac{2}{\beta c t}\right)^{v} \Gamma(v+1) I_{v}(\beta c t),
$$

where $I_{v}(x)=\sum_{k=0}^{\infty}(x / 2)^{2 k+v} / \Gamma(k+1) \Gamma(k+v+1)$ represents the modified Bessel function, with $x \in \mathbb{R}$ and $v \in \mathbb{R}$.

Proof. The proofs of (2.7) and (2.6) follow analogously to the those developed for (2.3) and (2.4), noting that

$$
I_{\nu}(x)=\frac{(x / 2)^{v}}{\Gamma(v+1 / 2) \Gamma(1 / 2)} \int_{0}^{\pi} \mathrm{e}^{x \cos \phi} \sin ^{2 v} \phi \mathrm{d} \phi
$$

with $\operatorname{Re}(v+1 / 2)>0$. This completes the proof.

The random motions obtained by setting $v=0$ and $v=1$ have a special role in this paper. Indeed, for $X_{0}(t), t>0$, and $X_{1}(t), t>0$, we are able to find explicit closed-form expressions 
for their characteristic and moment generating functions and the density laws. In order to distinguish these important particular cases from the general random model $X_{v}(t), v \geq 0$, we will indicate them in the rest of the paper with $X_{m}(t), m=0,1$. Moreover, we will use the following notation: $v_{0}=n / 2$ and $v_{1}=n+1$.

Theorem 2.3. For $X_{m}(t), t>0, m=0,1$, and $n \geq 1$, we have

$$
\begin{aligned}
& \mathrm{E}\left\{\mathrm{e}^{\mathrm{i} \alpha X_{m}(t)} \mid N(t)=n\right\}=\frac{\Gamma\left(v_{m}+1\right) 2^{v_{m}}}{(\alpha c t)^{v_{m}}} J_{v_{m}}(\alpha c t), \\
& \mathrm{E}\left\{\mathrm{e}^{\beta X_{m}(t)} \mid N(t)=n\right\}=\frac{\Gamma\left(v_{m}+1\right) 2^{v_{m}}}{(\beta c t)^{v_{m}}} I_{v_{m}}(\beta c t) .
\end{aligned}
$$

Proof. Starting from (2.3), we prove (2.8) for $m=0$. Indeed, we have

$$
\mathrm{E}\left\{\mathrm{e}^{\beta X_{0}(t)} \mid N(t)=n\right\}=\frac{n !}{t^{n}} \int_{0}^{t} \mathrm{~d} s_{1} \cdots \int_{s_{n-1}}^{t} \mathrm{~d} s_{n} \prod_{j=1}^{n+1} J_{0}\left(\alpha c\left(s_{j}-s_{j-1}\right)\right),
$$

and, by means of the approach developed in Kolesnik and Orsingher (2005) (see the proof of Theorem 1), we observe that the $n$-fold integral

$$
\int_{0}^{t} \mathrm{~d} s_{1} \cdots \int_{s_{n-1}}^{t} \mathrm{~d} s_{n} \prod_{j=1}^{n+1} J_{0}\left(\alpha c\left(s_{j}-s_{j-1}\right)\right)
$$

can be evaluated by recursively applying the following formula (see Gradshteyn and Ryzhik (1980, Equation 6.581(3))):

$$
\int_{0}^{a} x^{\mu}(a-x)^{v} J_{\mu}(x) J_{v}(a-x) \mathrm{d} x=\frac{\Gamma(\mu+1 / 2) \Gamma(v+1 / 2)}{\sqrt{2 \pi} \Gamma(\mu+v+1)} a^{\mu+v+1 / 2} J_{\mu+v+1 / 2}(a)
$$

with $\operatorname{Re}(\mu)>-\frac{1}{2}$ and $\operatorname{Re}(\nu)>-\frac{1}{2}$. Therefore, at the first step we have

$$
\begin{aligned}
\int_{s_{n-1}}^{t} & J_{0}\left(\alpha c\left(t-s_{n}\right)\right) J_{0}\left(\alpha c\left(s_{n}-s_{n-1}\right)\right) \mathrm{d} s_{n} \\
= & \frac{1}{\alpha c} \int_{0}^{\alpha c\left(t-s_{n-1}\right)} J_{0}(w) J_{0}\left(\alpha c\left(t-s_{n-1}\right)-w\right) \mathrm{d} w \\
= & \frac{1}{\alpha c} \frac{\Gamma^{2}(1 / 2)}{\sqrt{2 \pi} \Gamma(1)}\left(\alpha c\left(t-s_{n-1}\right)\right)^{1 / 2} J_{1 / 2}\left(\alpha c\left(t-s_{n-1}\right)\right),
\end{aligned}
$$

where we have used the change of variable $w=\alpha c\left(s_{n}-s_{n-1}\right)$.

At the second step we obtain

$$
\begin{aligned}
\frac{1}{\alpha c} & \frac{\Gamma^{2}(1 / 2)}{\sqrt{2 \pi} \Gamma(1)} \int_{s_{n-2}}^{t}\left(\alpha c\left(t-s_{n-1}\right)\right)^{1 / 2} J_{1 / 2}\left(\alpha c\left(t-s_{n-1}\right)\right) J_{0}\left(\alpha c\left(s_{n-1}-s_{n-2}\right)\right) \mathrm{d} s_{n-1} \\
& =\frac{1}{(\alpha c)^{2}} \frac{\Gamma^{2}(1 / 2)}{\sqrt{2 \pi} \Gamma(1)} \int_{0}^{\alpha c\left(t-s_{n-2}\right)}\left(\alpha c\left(t-s_{n-2}\right)-w\right)^{1 / 2} J_{0}(w) J_{1 / 2}\left(\alpha c\left(t-s_{n-2}\right)-w\right) \mathrm{d} w \\
& =\frac{1}{(\alpha c)^{2}} \frac{\Gamma^{3}(1 / 2)}{(\sqrt{2 \pi})^{2} \Gamma(3 / 2)}\left(\alpha c\left(t-s_{n-2}\right)\right) J_{1}\left(\alpha c\left(t-s_{n-2}\right)\right),
\end{aligned}
$$


where we have used the change of variable $w=\alpha c\left(s_{n-1}-s_{n-2}\right)$. Therefore, at the $(n-1)$ th step, we have

$$
\begin{aligned}
& \frac{1}{(\alpha c)^{n-1}} \frac{(\Gamma(1 / 2))^{n}}{(\sqrt{2 \pi})^{n-1} \Gamma(n / 2)} \int_{0}^{t}\left(\alpha c\left(t-s_{1}\right)\right)^{(n-1) / 2} J_{(n-1) / 2}\left(\alpha c\left(t-s_{1}\right)\right) J_{0}\left(\alpha c s_{1}\right) \mathrm{d} s_{1} \\
& =\frac{1}{(\alpha c)^{n}} \frac{(\Gamma(1 / 2))^{n}}{(\sqrt{2 \pi})^{n-1} \Gamma(n / 2)} \int_{0}^{\alpha c t}(\alpha c t-w)^{(n-1) / 2} J_{(n-1) / 2}(\alpha c t-w) J_{0}(w) \mathrm{d} w \\
& =\frac{1}{(\alpha c)^{n}} \frac{(\Gamma(1 / 2))^{n+1}}{(\sqrt{2 \pi})^{n} \Gamma((n+1) / 2)}(\alpha c t)^{n / 2} J_{n / 2}(\alpha c t),
\end{aligned}
$$

where we have used the change of variable $w=\alpha c s_{1}$.

Finally, by means of the duplication formula for gamma functions, we obtain

$$
\begin{aligned}
\mathrm{E}\left\{\mathrm{e}^{\mathrm{i} \alpha X_{0}(t)} \mid N(t)=n\right\} & =\frac{n !}{t^{n}} \frac{1}{(\alpha c)^{n}} \frac{(\Gamma(1 / 2))^{n+1}}{(\sqrt{2 \pi})^{n} \Gamma((n+1) / 2)}(\alpha c t)^{n / 2} J_{n / 2}(\alpha c t) \\
& =\frac{2^{n / 2} \Gamma(n / 2+1)}{(\alpha c t)^{n / 2}} J_{n / 2}(\alpha c t) .
\end{aligned}
$$

By taking into account the formula (see Gradshteyn and Ryzhik (1980, Equation 6.533(2)))

$$
\int_{0}^{a} \frac{J_{\mu}(x)}{x} \frac{J_{v}(a-x)}{a-x} \mathrm{~d} x=\left(\frac{1}{\mu}+\frac{1}{v}\right) \frac{J_{\mu+v}(a)}{a},
$$

with $\operatorname{Re}(\mu)>0$ and $\operatorname{Re}(\nu)>0$, it is possible to compute exactly the characteristic function

$$
\mathrm{E}\left\{\mathrm{e}^{\mathrm{i} \alpha X_{1}(t)} \mid N(t)=n\right\}=\frac{n !}{t^{n}} 2^{n+1} \int_{0}^{t} \mathrm{~d} s_{1} \cdots \int_{s_{n-1}}^{t} \mathrm{~d} s_{n} \prod_{j=1}^{n+1} \frac{J_{1}\left(\alpha c\left(s_{j}-s_{j-1}\right)\right)}{\alpha c\left(s_{j}-s_{j-1}\right)}
$$

emerging from (2.3) for $v=1$. Hence, as above, we recursively apply (2.11), and at the first step we have

$$
\int_{s_{n-1}}^{t} \frac{J_{1}\left(\alpha c\left(s_{n}-s_{n-1}\right)\right)}{\alpha c\left(s_{n}-s_{n-1}\right)} \frac{J_{1}\left(\alpha c\left(t-s_{n}\right)\right)}{\alpha c\left(t-s_{n}\right)} \mathrm{d} s_{n}=\frac{2}{\alpha c} \frac{J_{2}\left(\alpha c\left(t-s_{n-1}\right)\right)}{\alpha c\left(t-s_{n-1}\right)} .
$$

The integral with respect to $s_{n-1}$ becomes

$$
\frac{2}{\alpha c} \int_{s_{n-2}}^{t} \frac{J_{1}\left(\alpha c\left(s_{n-1}-s_{n-2}\right)\right)}{\alpha c\left(s_{n-1}-s_{n-2}\right)} \frac{J_{2}\left(\alpha c\left(t-s_{n-1}\right)\right)}{\alpha c\left(t-s_{n-1}\right)} \mathrm{d} s_{n-1}=\frac{3}{(\alpha c)^{2}} \frac{J_{3}\left(\alpha c\left(t-s_{n-2}\right)\right)}{\alpha c\left(t-s_{n-2}\right)},
$$

and then in the last step we have

$$
\frac{n}{(\alpha c)^{n-1}} \int_{s_{1}}^{t} \frac{J_{1}\left(\alpha c s_{1}\right)}{\alpha c s_{1}} \frac{J_{n}\left(\alpha c\left(t-s_{1}\right)\right)}{\alpha c\left(t-s_{1}\right)} \mathrm{d} s_{1}=\frac{n+1}{(\alpha c)^{n}} \frac{J_{n+1}(\alpha c t)}{\alpha c t} .
$$

Therefore, the characteristic function for $X_{1}(t), t>0$, immediately follows.

In order to show (2.9), we need to prove that

$$
\int_{0}^{a} x^{\mu}(a-x)^{v} I_{\mu}(x) I_{\nu}(a-x) \mathrm{d} x=\frac{\Gamma(\mu+1 / 2) \Gamma(v+1 / 2)}{\sqrt{2 \pi} \Gamma(\mu+v+1)} a^{\mu+v+1 / 2} I_{\mu+v+1 / 2}(a)
$$


for $\operatorname{Re}(\mu)>-\frac{1}{2}$ and $\operatorname{Re}(v)>-\frac{1}{2}$, and

$$
\int_{0}^{a} \frac{I_{\mu}(x)}{x} \frac{I_{\nu}(a-x)}{a-x} \mathrm{~d} x=\left(\frac{1}{\mu}+\frac{1}{v}\right) a^{-1} I_{\mu+v}(a)
$$

for $\operatorname{Re}(\mu)>0$ and $\operatorname{Re}(\nu)>0$. Result (2.13) can be proved as follows. Since (see Gradshteyn and Ryzhik (1980, Equation (46), p. 1145))

$$
\int_{0}^{\infty} \mathrm{e}^{-b x} x^{\mu} I_{\mu}(x) \mathrm{d} x=\frac{2^{\mu} \Gamma(\mu+1 / 2)}{\sqrt{\pi}\left(b^{2}-1\right)^{\mu+1 / 2}}, \quad \operatorname{Re}(\mu)>-\frac{1}{2}, \operatorname{Re}(b)>1,
$$

we have

$$
\begin{aligned}
\int_{0}^{\infty} & \mathrm{e}^{-b a} \mathrm{~d} a \int_{0}^{a} x^{\mu}(a-x)^{v} I_{\mu}(x) I_{v}(a-x) \mathrm{d} x \\
& =\int_{0}^{\infty} \mathrm{e}^{-b x} x^{\mu} I_{\mu}(x) \mathrm{d} x \int_{0}^{\infty} \mathrm{e}^{-b y} y^{v} I_{\nu}(y) \mathrm{d} y \\
& =2^{\mu+v} \pi^{-1} \frac{\Gamma(\mu+1 / 2) \Gamma(v+1 / 2)}{\left(b^{2}-1\right)^{\mu+v+1}} \\
& =\frac{\Gamma(\mu+1 / 2) \Gamma(v+1 / 2)}{\sqrt{2 \pi} \Gamma(\mu+v+1)} \int_{0}^{\infty} \mathrm{e}^{-b a} a^{\mu+v+1 / 2} I_{\mu+v+1 / 2}(a) \mathrm{d} a .
\end{aligned}
$$

Furthermore, since (see Gradshteyn and Ryzhik (1980, Equation (48), p. 1146))

$$
\int_{0}^{\infty} \mathrm{e}^{-b x} x^{-1} I_{\mu}(x) \mathrm{d} x=\frac{1}{\mu\left(b+\left(b^{2}-1\right)^{1 / 2}\right)^{\mu}}, \quad \operatorname{Re}(\mu)>0, \operatorname{Re}(b)>1,
$$

using similar steps to those used to prove (2.13), it is possible to show that (2.14) holds.

In conclusion, by means of (2.13) and (2.14), and by carrying out the same procedure used for the proof of (2.8), the proof of (2.9) immediately follows. This completes the proof.

In the next theorem we present the conditional probability distributions of $X_{0}(t), t>0$, and $X_{1}(t), t>0$.

Theorem 2.4. The following conditional densities hold:

$$
\begin{aligned}
& p_{0}^{v}(x, t)=\frac{\mathrm{P}\left(X_{v}(t) \in \mathrm{d} x \mid N(t)=0\right)}{\mathrm{d} x}=\frac{\Gamma(v) \Gamma(v+1)}{2 \pi \Gamma(2 v)}\left(\frac{2}{c t}\right)^{2 v}\left(c^{2} t^{2}-x^{2}\right)^{v-1 / 2}, \\
& p_{n}^{m}(x, t)=\frac{\mathrm{P}\left(X_{m}(t) \in \mathrm{d} x \mid N(t)=n\right)}{\mathrm{d} x}=\frac{\Gamma\left(v_{m}\right) \Gamma\left(v_{m}+1\right)}{2 \pi \Gamma\left(2 v_{m}\right)}\left(\frac{2}{c t}\right)^{2 v_{m}}\left(c^{2} t^{2}-x^{2}\right)^{v_{m}-1 / 2},
\end{aligned}
$$

with $n \geq 1,|x|<c t$, and $m=0,1$.

Proof. We prove only (2.15) for $m=0$, because the other results follow by means of similar steps. Instead of inverting (2.8), we show that the characteristic function of the probability 
distribution $p_{n}^{0}(x, t)$ corresponds to (2.8) for $m=0$. Therefore, we have

$$
\begin{aligned}
\mathrm{E}\left\{\mathrm{e}^{\mathrm{i} \alpha} X_{0}(t)\right. & \mid N(t)=n\} \\
& =\frac{\Gamma(n / 2) \Gamma(n / 2+1)}{2 \pi \Gamma(n)}\left(\frac{2}{c t}\right)^{n} \int_{-c t}^{c t} \mathrm{e}^{\mathrm{i} \alpha x}\left(c^{2} t^{2}-x^{2}\right)^{(n-1) / 2} \mathrm{~d} x \\
& =\frac{\Gamma(n / 2) \Gamma(n / 2+1)}{\pi \Gamma(n)}\left(\frac{2}{c t}\right)^{n} \int_{0}^{c t} \cos (\alpha x)\left(c^{2} t^{2}-x^{2}\right)^{(n-1) / 2} \mathrm{~d} x \\
& =\frac{\Gamma(n / 2) \Gamma(n / 2+1)}{\pi \Gamma(n)}\left(\frac{2}{c t}\right)^{n} \sum_{k=0}^{\infty}(-1)^{k} \frac{\alpha^{2 k}}{(2 k) !} \int_{0}^{c t} x^{2 k}\left(c^{2} t^{2}-x^{2}\right)^{(n-1) / 2} \mathrm{~d} x \\
& =\frac{\Gamma(n / 2) \Gamma(n / 2+1) 2^{n-1}}{\pi \Gamma(n)} \sum_{k=0}^{\infty}(-1)^{k} \frac{(\alpha c t)^{2 k}}{(2 k) !} \int_{0}^{1} y^{k-1 / 2}(1-y)^{(n-1) / 2} \mathrm{~d} y \\
& =\frac{\Gamma(n / 2) \Gamma(n / 2+1) \Gamma((n+1) / 2) 2^{n-1}}{\pi(n)} \sum_{k=0}^{\infty}(-1)^{k} \frac{(\alpha c t)^{2 k}}{(2 k) !} \frac{\Gamma(k+1 / 2)}{\Gamma(k+n / 2+1)} \\
= & \frac{\Gamma(n / 2) \Gamma(n / 2+1) \Gamma(n) \sqrt{\pi}}{\pi \Gamma(n) \Gamma(n / 2)} \sum_{k=0}^{\infty}(-1)^{k} \frac{(\alpha c t)^{2 k}}{(2 k) !} \frac{\Gamma(2 k) \sqrt{\pi} 2^{1-2 k}}{\Gamma(k) \Gamma(k+n / 2+1)} \\
= & \Gamma\left(\frac{n}{2}+1\right) \sum_{k=0}^{\infty}(-1)^{k}\left(\frac{\alpha c t}{2}\right)^{2 k} \frac{1}{\Gamma(k+1) \Gamma(k+n / 2+1)} \\
= & \frac{\Gamma(n / 2+1) 2^{n / 2}}{(\alpha c t)^{n / 2}} J_{n / 2}(\alpha c t) .
\end{aligned}
$$

This completes the proof.

Remark 2.1. Theorem 2.4 permits us to point out the connection between $X_{0}(t), X_{1}(t)$, and the random flights studied in Stadje (1987), Kolesnik and Orsingher (2005), and De Gregorio and Orsingher (2007). Indeed, by setting $v=0$ in $f_{v}(\theta)$, we again obtain the uniform distribution on a unit semicircle, and (as expected) $X_{0}(t)$ represents the projection onto the $x$-axis of a planar random flight. Then

$$
p_{n}^{0}(x, t)=\frac{\Gamma(n / 2) \Gamma(n / 2+1)}{2 \pi \Gamma(n)}\left(\frac{2}{c t}\right)^{n}\left(c^{2} t^{2}-x^{2}\right)^{(n-1) / 2}
$$

corresponds to the distribution (4.4) of Orsingher and De Gregorio (2007). For $v=1$, the probability distribution

$$
p_{n}^{1}(x, t)=\frac{\Gamma(n+1) \Gamma(n+2)}{2 \pi \Gamma(2 n+2)}\left(\frac{2}{c t}\right)^{2 n+2}\left(c^{2} t^{2}-x^{2}\right)^{n+1 / 2}
$$

coincides with the density obtained by means of the projection of a random flight in $\mathbb{R}^{4}$ onto the real line (see Equation (4.1c) of Orsingher and De Gregorio (2007)). In other words, the shadow on $\mathbb{R}$ of a four-dimensional random flight is perceived by an observer located on the real line as a slowed down motion. Therefore, in distribution, we have

$$
X_{1}(t) \stackrel{\mathrm{D}}{=} c \sum_{j=1}^{N(t)+1}\left(s_{j}-s_{j-1}\right) \sin \theta_{1, j} \sin \theta_{2, j} \sin \phi,
$$

where $\left(\theta_{1, j}, \theta_{2, j}, \phi\right)$ is uniformly distributed on the four-dimensional hypersphere. 
Remark 2.2. From Theorem 2.4 we observe that $p_{0}^{v}(x, t)=p_{n}^{m}(x, t)$ if and only if $v=v_{m}$. This means that $X_{0}(t)$ (absence of friction) and $X_{1}(t)$ are equivalent in distribution to a random model representing a particle slowly moving with the same speed until $t$.

By averaging $p_{n}^{0}(x, t)$ and $p_{n}^{1}(x, t)$ with $\mathrm{P}\{N(t)=n\}=\mathrm{e}^{-\lambda t}(\lambda t)^{n} / n !, n \geq 0$, the unconditional densities of $X_{0}(t)$ and $X_{1}(t)$ yield

$$
\begin{aligned}
p^{0}(x, t)= & \frac{\lambda \mathrm{e}^{-\lambda t}}{2 c} \sum_{k=0}^{\infty}\left(\frac{\lambda}{2 c} \sqrt{c^{2} t^{2}-x^{2}}\right)^{k-1} \frac{1}{\Gamma^{2}((k+1) / 2)} \\
= & \frac{\lambda \mathrm{e}^{-\lambda t}}{2 c}\left[I_{0}\left(\frac{\lambda}{c} \sqrt{c^{2} t^{2}-x^{2}}\right)+\mathcal{L}_{0}\left(\frac{\lambda}{c} \sqrt{c^{2} t^{2}-x^{2}}\right)\right] \\
& +\frac{\mathrm{e}^{-\lambda t}}{\pi \sqrt{c^{2} t^{2}-x^{2}}}, \quad|x|<c t,
\end{aligned}
$$

where

$$
\mathcal{L}_{\mu}(x)=\sum_{k=0}^{\infty} \frac{(x / 2)^{2 k+\mu+1}}{\Gamma(k+3 / 2) \Gamma(k+\mu+3 / 2)}, \quad x \in \mathbb{R}, \mu \in \mathbb{R},
$$

is the modified Struve function, and

$$
\begin{aligned}
p^{1}(x ; t) & =\frac{\mathrm{e}^{-\lambda t}}{c \sqrt{\lambda \pi t^{3}}} \sum_{k=0}^{\infty}\left(\frac{\lambda}{c^{2} t}\left(c^{2} t^{2}-x^{2}\right)\right)^{k+1 / 2} \frac{k+1}{\Gamma(k+3 / 2)} \\
& =\frac{\mathrm{e}^{-\lambda t} \sqrt{c^{2} t^{2}-x^{2}}}{(c t)^{2} \sqrt{\pi}}\left\{E_{1,1 / 2}\left(\frac{\lambda}{c^{2} t}\left(c^{2} t^{2}-x^{2}\right)\right)+\frac{1}{2} E_{1,3 / 2}\left(\frac{\lambda}{c^{2} t}\left(c^{2} t^{2}-x^{2}\right)\right)\right\}
\end{aligned}
$$

for $|x|<c t$, where $E_{\alpha, \beta}(x)=\sum_{k=0}^{\infty} x^{k} / \Gamma(\alpha x+\beta), x \in \mathbb{R}, \alpha, \beta>0$, is the Mittag-Leffler function.

Note that the first term in (2.16), that is, $I_{0}\left(\lambda \sqrt{c^{2} t^{2}-x^{2}} / c\right)$, is equal to that given in the absolutely continuous component of the law of a telegraph process (see Orsingher (1990)), that is,

$$
\frac{\mathrm{e}^{-\lambda t}}{2 c}\left[\lambda I_{0}\left(\frac{\lambda}{c} \sqrt{c^{2} t^{2}-x^{2}}\right)+\frac{\partial}{\partial t} I_{0}\left(\frac{\lambda}{c} \sqrt{c^{2} t^{2}-x^{2}}\right)\right],
$$

while the derivative with respect to the time of the Bessel function is replaced by the modified Struve function $\mathcal{L}_{0}\left(\lambda \sqrt{c^{2} t^{2}-x^{2}} / c\right)$.

We are able to provide the first two moments of $X_{v}(t)$ by applying the results contained in Section 3 of Stadje and Zacks (2004). Therefore, for fixed $n \geq 0$, the mean value is given by

$$
\begin{aligned}
\mathrm{E}\left\{X_{v}(t) \mid N(t)=n\right\} & =c t \mathrm{E}\{\cos \theta\} \\
& =\frac{c t \Gamma(v+1)}{\sqrt{\pi} \Gamma(v+1 / 2)} \int_{0}^{\pi} \cos \theta \sin ^{2 v} \theta \mathrm{d} \theta \\
& =\frac{c t \Gamma(v+1)}{\sqrt{\pi} \Gamma(v+1 / 2)}\left(\int_{0}^{\pi / 2} \cos \theta \sin ^{2 v} \theta \mathrm{d} \theta-\int_{0}^{\pi / 2} \sin \theta \cos ^{2 v} \theta \mathrm{d} \theta\right) \\
& =0,
\end{aligned}
$$


where in the last step we used the well-known integral

$$
\int_{0}^{\pi / 2} \sin ^{a} \theta \cos ^{b} \theta \mathrm{d} \theta=\frac{1}{2} \frac{\Gamma((a+1) / 2) \Gamma((b+1) / 2)}{\Gamma((a+b) / 2+1)}, \quad \operatorname{Re}(a)>-1, \operatorname{Re}(b)>-1 .
$$

Remark 2.3. The mean value can also be derived from Theorem 2.1 (or, equivalently, from Theorem 2.2). It is clear that

$$
\left.\frac{J_{v}\left(\alpha c\left(s_{j}-s_{j-1}\right)\right)}{\left(\alpha c\left(s_{j}-s_{j-1}\right)\right)^{v}}\right|_{\alpha=0}=\frac{1}{2^{v} \Gamma(v+1)}
$$

while it is not hard to prove that

$$
\frac{\mathrm{d}}{\mathrm{d} \alpha} \frac{J_{v}\left(\alpha c\left(s_{j}-s_{j-1}\right)\right)}{\left(\alpha c\left(s_{j}-s_{j-1}\right)\right)^{v}}=\sum_{k=0}^{\infty} \frac{k}{k ! \Gamma(k+v+1)}\left(\frac{\alpha c\left(s_{j}-s_{j-1}\right)}{2}\right)^{2 k+v-1} \frac{c\left(s_{j}-s_{j-1}\right)}{\left(\alpha c\left(s_{j}-s_{j-1}\right)\right)^{v}},
$$

which calculated at $\alpha=0$ is equal to 0 . Then

$$
\mathrm{E}\left\{X_{v}(t) \mid N(t)=n\right\}=\left.\mathrm{i}^{-1} \frac{\mathrm{d}}{\mathrm{d} \alpha} \mathrm{E}\left\{\mathrm{e}^{\mathrm{i} \alpha X_{v}(t)} \mid N(t)=n\right\}\right|_{\alpha=0}=0 .
$$

For the second moment, we have (see Stadje and Zacks (2004, p. 669))

$$
\begin{aligned}
\mathrm{E}\left\{X_{v}^{2}(t) \mid N(t)=n\right\} & =\frac{2}{n+2} c^{2} t^{2} \mathrm{E}\left\{\cos ^{2} \theta\right\} \\
& =\frac{2}{n+2} c^{2} t^{2} \frac{\Gamma(v+1)}{\sqrt{\pi} \Gamma(v+1 / 2)} \int_{0}^{\pi} \cos ^{2} \theta \sin ^{2 v} \theta \mathrm{d} \theta \\
& =\frac{2}{n+2} c^{2} t^{2} \frac{\Gamma(v+1) \Gamma(3 / 2)}{\sqrt{\pi} \Gamma(v+2)} \\
& =\frac{c^{2} t^{2}}{(v+1)(n+2)}
\end{aligned}
$$

and

$$
\begin{aligned}
\mathrm{E}\left\{X_{v}^{2}(t)\right\} & =\mathrm{e}^{-\lambda t} \frac{(c t)^{2}}{v+1} \sum_{n=0}^{\infty} \frac{(\lambda t)^{n}}{n !(n+2)} \\
& =\mathrm{e}^{-\lambda t} \frac{(c t)^{2}}{v+1} \sum_{n=0}^{\infty} \frac{(\lambda t)^{n}(n+1)}{(n+2) !} \\
& =\mathrm{e}^{-\lambda t} \frac{(c t)^{2}}{v+1}\left(E_{1,2}(\lambda t)-E_{1,3}(\lambda t)\right) \\
& =\frac{(c t)^{2}}{v+1} \frac{1}{\lambda t}\left(1-\frac{1-\mathrm{e}^{-\lambda t}}{\lambda t}\right),
\end{aligned}
$$

where in the last step we used the following relationships: $E_{1,2}(x)=\left(\mathrm{e}^{x}-1\right) / x$ and $E_{1,3}=$ $\left(\mathrm{e}^{x}-1-x\right) / x^{2}$. As expected, if $v$ increases, the action of the friction is stronger and the value of $\mathrm{E}\left\{X_{v}^{2}(t)\right\}$ tends to decrease. Indeed, for growing values of $v$, the particle maintains a position close to the starting point, so that the probability distribution of $X_{v}(t)$ will be less sparse. 
Furthermore, it is not difficult to show that

$$
\mathrm{E}\left\{X_{v}^{p}(t) \mid N(t)=0\right\}=\int_{-c t}^{c t} x^{p} p_{0}^{v}(x, t) \mathrm{d} x= \begin{cases}0 & p \text { is odd } \\ \frac{\Gamma(v+1) \Gamma((p+1) / 2)}{\sqrt{\pi} \Gamma(p / 2+v+1)}(c t)^{p} & p \text { is even. }\end{cases}
$$

For $v=0,1$, we present the following result.

Theorem 2.5. The pth moments of $X_{0}(t), t>0$, and $X_{1}(t), t>0$, are respectively given by

$$
\begin{aligned}
\mathrm{E}\left\{X_{0}^{p}(t)\right\}= & \mathrm{e}^{-\lambda t}\left(\frac{2}{\lambda t}\right)^{(p-1) / 2}(c t)^{p} \Gamma\left(\frac{p+1}{2}\right)\left\{I_{(p+1) / 2}(\lambda t)+\mathcal{L}_{(p+1) / 2}(\lambda t)\right\} \\
& +\frac{\mathrm{e}^{-\lambda t}(c t)^{p} \Gamma((p+1) / 2)}{\sqrt{\pi} \Gamma(p / 2+1)}, \\
\mathrm{E}\left\{X_{1}^{p}(t)\right\}= & \frac{\mathrm{e}^{-\lambda t}}{\sqrt{\pi}} \Gamma\left(\frac{p+1}{2}\right)(c t)^{p}\left(E_{1, p / 2+1}(\lambda t)-\frac{p}{2} E_{1, p / 2+2}(\lambda t)\right),
\end{aligned}
$$

if $p$ is even, whilst $\mathrm{E}\left\{X_{0}^{p}(t)\right\}=\mathrm{E}\left\{X_{1}^{p}(t)\right\}=0$ if $p$ is odd.

Proof. Let $p$ be even. Then

$$
\begin{aligned}
\mathrm{E}\left\{X_{0}^{p}(t)\right\} & =\frac{\lambda \mathrm{e}^{-\lambda t}}{c} \sum_{k=0}^{\infty}\left(\frac{\lambda}{2 c}\right)^{k-1} \frac{1}{\Gamma^{2}((k+1) / 2)} \int_{0}^{c t} x^{p}\left(c^{2} t^{2}-x^{2}\right)^{(k-1) / 2} \mathrm{~d} x \\
& =\frac{\lambda \mathrm{e}^{-\lambda t}}{2 c} \sum_{k=0}^{\infty}\left(\frac{\lambda}{2 c}\right)^{k-1} \frac{(c t)^{p+k}}{\Gamma^{2}((k+1) / 2)} \int_{0}^{1} y^{(p+1) / 2-1}(1-y)^{(k+1) / 2-1} \mathrm{~d} y \\
& =\frac{\lambda \mathrm{e}^{-\lambda t}}{2 c}(c t)^{p} \Gamma\left(\frac{p+1}{2}\right) \sum_{k=0}^{\infty}\left(\frac{\lambda}{2 c}\right)^{k-1} \frac{(c t)^{k}}{\Gamma((k+1) / 2) \Gamma((k+p) / 2+1)}
\end{aligned}
$$

where we have used the change of variable $x=c t \sqrt{y}$.

Now, splitting the above sum into even and the odd elements, we obtain

$$
\begin{aligned}
\mathrm{E}\left\{X_{0}^{p}(t)\right\}= & \frac{\lambda \mathrm{e}^{-\lambda t}}{2 c}(c t)^{p} \Gamma\left(\frac{p+1}{2}\right)\left\{\sum_{k=0}^{\infty}\left(\frac{\lambda}{2 c}\right)^{2 k} \frac{(c t)^{2 k+1}}{\Gamma(k+1) \Gamma(k+(p+1) / 2+1)}\right. \\
& \left.+\sum_{k=0}^{\infty}\left(\frac{\lambda}{2 c}\right)^{2 k-1} \frac{(c t)^{2 k}}{\Gamma(k+1 / 2) \Gamma(k+p / 2+1)}\right\} \\
= & \frac{\lambda \mathrm{e}^{-\lambda t}}{2 c}(c t)^{p} \Gamma\left(\frac{p+1}{2}\right) \\
& \times\left\{c t \sum_{k=0}^{\infty}\left(\frac{\lambda t}{2}\right)^{2 k} \frac{1}{\Gamma(k+1) \Gamma(k+(p+1) / 2+1)}+\left(\frac{\lambda}{2 c}\right)^{-1} \frac{1}{\sqrt{\pi} \Gamma(p / 2+1)}\right. \\
& \left.\quad+\left(\frac{\lambda}{2 c}\right)^{-1} \sum_{k=1}^{\infty}\left(\frac{\lambda t}{2}\right)^{2 k} \frac{1}{\Gamma(k+1 / 2) \Gamma(k+p / 2+1)}\right\}
\end{aligned}
$$




$$
\begin{aligned}
& =\frac{\lambda \mathrm{e}^{-\lambda t}}{2 c}(c t)^{p} \Gamma\left(\frac{p+1}{2}\right) \\
& \quad \times\left\{c t \sum_{k=0}^{\infty}\left(\frac{\lambda t}{2}\right)^{2 k} \frac{1}{\Gamma(k+1) \Gamma(k+(p+1) / 2+1)}+\left(\frac{\lambda}{2 c}\right)^{-1} \frac{1}{\sqrt{\pi} \Gamma(p / 2+1)}\right. \\
& \left.\quad+\left(\frac{\lambda}{2 c}\right)^{-1} \sum_{k=0}^{\infty}\left(\frac{\lambda t}{2}\right)^{2 k+2} \frac{1}{\Gamma(k+3 / 2) \Gamma(k+(p+1) / 2+3 / 2)}\right\} \\
& =\frac{\lambda \mathrm{e}^{-\lambda t}}{2 c}(c t)^{p} \Gamma\left(\frac{p+1}{2}\right)\left\{c t\left(\frac{\lambda t}{2}\right)^{-(p+1) / 2}\left(I_{(p+1) / 2}(\lambda t)+\mathcal{L}_{(p+1) / 2}(\lambda t)\right)\right. \\
& \\
& \left.\quad+\left(\frac{\lambda}{2 c}\right)^{-1} \frac{1}{\sqrt{\pi} \Gamma(p / 2+1)}\right\} .
\end{aligned}
$$

For $X_{1}(t)$, we have

$$
\begin{aligned}
\mathrm{E}\left\{X_{1}^{p}(t)\right\} & =\frac{2 \mathrm{e}^{-\lambda t}}{c \sqrt{\lambda \pi t^{3}}} \sum_{k=0}^{\infty}\left(\frac{\lambda}{c^{2} t}\right)^{k+1 / 2} \frac{k+1}{\Gamma(k+3 / 2)} \int_{0}^{c t} x^{p}\left(c^{2} t^{2}-x^{2}\right)^{k+1 / 2} \mathrm{~d} x \\
& =\frac{\mathrm{e}^{-\lambda t}}{c \sqrt{\lambda \pi t^{3}}} \sum_{k=0}^{\infty}\left(\frac{\lambda}{c^{2} t}\right)^{k+1 / 2} \frac{k+1}{\Gamma(k+3 / 2)}(c t)^{p+2 k+2} \int_{0}^{1} y^{(p-1) / 2}(1-y)^{k+1 / 2} \mathrm{~d} y \\
& =\frac{\mathrm{e}^{-\lambda t}}{c \sqrt{\lambda \pi t^{3}}}\left(\frac{\lambda}{c^{2} t}\right)^{1 / 2} \Gamma\left(\frac{p+1}{2}\right)(c t)^{p+2} \sum_{k=0}^{\infty}(\lambda t)^{k} \frac{k+1}{\Gamma(k+p / 2+2)} \\
& =\frac{\mathrm{e}^{-\lambda t}}{\sqrt{\pi}} \Gamma\left(\frac{p+1}{2}\right)(c t)^{p} \sum_{k=0}^{\infty}(\lambda t)^{k} \frac{k+p / 2+1-p / 2}{\Gamma(k+p / 2+2)} \\
& =\frac{\mathrm{e}^{-\lambda t}}{\sqrt{\pi}} \Gamma\left(\frac{p+1}{2}\right)(c t)^{p}\left(E_{1, p / 2+1}(\lambda t)-\frac{p}{2} E_{1, p / 2+2}(\lambda t)\right)
\end{aligned}
$$

where we have used the change of variable $x=c t \sqrt{y}$.

If $p$ is odd, it immediately follows that $\mathrm{E}\left\{X_{0}^{p}(t)\right\}=\mathrm{E}\left\{X_{1}^{p}(t)\right\}=0$. This complete the proof.

Remark 2.4. From (2.19) we immediately obtain expression (2.18) again for $v=1$, by setting $p=2$. For $\mathrm{E}\left\{X_{0}^{2}(t)\right\}$, starting from (2.20) and using the duplication formula for gamma functions, we have

$$
\begin{aligned}
\mathrm{E}\left\{X_{0}^{2}(t)\right\} & =\frac{\lambda \mathrm{e}^{-\lambda t}}{2 c}(c t)^{2} \Gamma\left(\frac{3}{2}\right) \sum_{k=0}^{\infty}\left(\frac{\lambda}{2 c}\right)^{k-1} \frac{(c t)^{k}}{\Gamma((k+1) / 2) \Gamma(k / 2+2)} \\
& =\frac{\mathrm{e}^{-\lambda t}(c t)^{2}}{2} \sum_{k=0}^{\infty} \frac{(\lambda t)^{k}}{\Gamma(k+1)} \frac{\Gamma(k / 2+1)}{\Gamma(k / 2+2)} \\
& =\mathrm{e}^{-\lambda t}(c t)^{2} \sum_{k=0}^{\infty} \frac{(\lambda t)^{k}}{\Gamma(k+1)(k+2)} \\
& =\mathrm{e}^{-\lambda t}(c t)^{2} \sum_{k=0}^{\infty} \frac{(\lambda t)^{k}(k+2-1)}{\Gamma(k+1)(k+1)(k+2)} \\
& =\mathrm{e}^{-\lambda t}(c t)^{2}\left(E_{1,2}(\lambda t)-E_{1,3}(\lambda t)\right),
\end{aligned}
$$

which coincides with (2.18) for $v=0$. 
Remark 2.5. Since, for $x>0$ and $\mu \geq 0, I_{\mu}(x), \mathcal{L}_{\mu}(x)$, and $E_{1, \mu}$ are monotone increasing functions as $x \rightarrow \infty$, the following approximations hold as $x \rightarrow 0$ :

$$
I_{\mu}(x) \sim \frac{x^{\mu}}{2^{\mu} \Gamma(\mu+1)}, \quad \mathcal{L}_{\mu}(x) \sim \frac{x^{\mu+1}}{\sqrt{\pi} 2^{\mu} \Gamma(\mu+3 / 2)}, \quad E_{1, \beta}(x) \sim \frac{1}{\Gamma(\beta)} .
$$

Therefore, for $\lambda t \rightarrow 0$, we have

$$
\begin{aligned}
\mathrm{E}\left\{X_{0}^{p}(t)\right\} \sim \mathrm{e}^{-\lambda t}(c t)^{p} \Gamma\left(\frac{p+1}{2}\right) & \left(\frac{\lambda t}{2} \frac{1}{\Gamma((p+1) / 2+1)}+\frac{(\lambda t)^{2}}{2 \sqrt{\pi}} \frac{1}{\Gamma((p+1) / 2+3 / 2)}\right. \\
& \left.+\frac{1}{\sqrt{\pi} \Gamma(p / 2+1)}\right), \\
\mathrm{E}\left\{X_{1}^{p}(t)\right\} \sim & \frac{\mathrm{e}^{-\lambda t}}{\sqrt{\pi}} \Gamma\left(\frac{p+1}{2}\right)(c t)^{p} \frac{1}{\Gamma(p / 2+2)} .
\end{aligned}
$$

\section{Stochastic motions with randomly varying time}

So far, we have analyzed a random motion $X_{v}(t)$ evolving up to a nonrandom time $t>0$, and derived its probability distribution in two particular cases. In this section we focus our attention on the random motion $X_{m}(t), m=0,1$, defined as in (2.2), with randomly varying time.

In order to develop our analysis, we take into account families of random times which include some well-known random variables. In particular, by considering Bessel and gamma processes as random times, we are able to include a wide range of probability distributions often used to model the time in many theoretical and real situations. Clearly, every random variable successively used as a random clock will be supposed to be independent from $X_{m}(t), t>0$, and $N(t), t>0$. Therefore, we analyze the effect due to the composition of these random times with the random motion $X_{m}(t), t>0$, on the related densities.

\subsection{Random times involving Brownian motions}

Let us consider a Bessel process starting from 0, i.e.

$$
B_{d}(t)=\sqrt{\sum_{i=1}^{d} W_{i}^{2}(t)}, \quad t>0, d \geq 1
$$

where the $W_{i}(t)$ s are independent standard Brownian motions. It is well known that the probability law of $B_{d}(t), t>0$, is equal to

$$
f_{d}(r)=\frac{1}{\Gamma(d / 2)} \frac{r^{d-1}}{2^{d / 2-1} t^{d / 2}} \mathrm{e}^{-r^{2} / 2 t}, \quad r>0 .
$$

At time $t$, we deal with the random motion $X_{m}(t), t>0, m=0,1$, having Bessel random time $B_{d}(t), t>0\left(X_{m}(t)\right.$ and $B_{d}(t)$ are assumed to be independent). Therefore, the change in velocity of $X_{m}\left(B_{d}(t)\right), t>0$, is governed by $N\left(B_{d}(t)\right), t>0$, i.e. a homogeneous Poisson process $N(t)$ with the Bessel time having the probability distribution

$$
\mathrm{P}\left\{N\left(B_{d}(t)\right)=n\right\}=\int_{0}^{\infty} \mathrm{P}\{N(s)=n\} f_{d}(s) \mathrm{d} s, \quad n \geq 0 .
$$


Since $X_{m}\left(B_{d}(t)\right)$ is located inside $\left(-c B_{d}(t), c B_{d}(t)\right)$, its support is the whole real line. Recalling that $v_{0}=n / 2$ and $v_{1}=n+1$, and by indicating with $B(a, b)=\Gamma(a) \Gamma(b) / \Gamma(a+b)$, $a>0, b>0$, the beta function, we are able to provide the following theorem.

Theorem 3.1. Given $N\left(B_{d}(t)\right)=n$, with $n \geq 1$, such that $v_{m}>d / 2-1$, we have the following conditional probability distribution:

$$
\begin{aligned}
& \mathrm{P}\left\{X_{m}\left(B_{d}(t)\right) \in \mathrm{d} x \mid N\left(B_{d}(t)\right)=n\right\} \\
& \quad=\frac{\mathrm{d} x}{B\left(d / 2, v_{m}-d / 2+1\right)} \int_{0}^{1} w^{d / 2-1}(1-w)^{v_{m}-d / 2} \frac{\mathrm{e}^{-x^{2} / 2 c^{2} t w}}{\sqrt{2 \pi t w} c} \mathrm{~d} w
\end{aligned}
$$

with $x \in \mathbb{R}$ and $m=0,1$.

Proof. By using a similar approach to that adopted in Beghin and Orsingher (2009), we can write

$$
\begin{aligned}
& \mathrm{P}\left\{X_{m}\left(B_{d}(t)\right) \in \mathrm{d} x \mid N\left(B_{d}(t)\right)=n\right\} \\
&= \int_{0}^{\infty} \mathrm{P}\left\{X_{m}\left(B_{d}(t)\right) \in \mathrm{d} x \mid N\left(B_{d}(t)\right)=n, B_{d}(t)=s\right\} \mathrm{P}\left\{B_{d}(t) \in \mathrm{d} s\right\} \\
&= \int_{0}^{\infty} \mathrm{P}\left\{X_{m}\left(B_{d}(t)\right) \in \mathrm{d} x \mid N(s)=n\right\} \mathrm{P}\left\{B_{d}(t) \in \mathrm{d} s\right\} \\
&= \frac{\mathrm{d} x}{2 \pi} \frac{\Gamma\left(v_{m}+1\right) \Gamma\left(v_{m}\right)}{\Gamma\left(2 v_{m}\right)} \frac{1}{\Gamma(d / 2) 2^{d / 2-1} t^{d / 2}} \\
& \times \int_{0}^{\infty}\left(\frac{2}{c s}\right)^{2 v_{m}}\left(c^{2} s^{2}-x^{2}\right)^{v_{m}-1 / 2} \mathbf{1}_{\{|x|<c s\}} s^{d-1} \mathrm{e}^{-s^{2} / 2 t} \mathrm{~d} s .
\end{aligned}
$$

Therefore, by means of Theorem 2.3 and, for any $n \geq 1$ such that $v_{m}>d / 2-1$, we are able to explicit the Fourier transform of $X_{m}\left(B_{d}(t)\right), t>0$, as follows:

$$
\begin{aligned}
\mathrm{E}\left\{\mathrm{e}^{\mathrm{i} \alpha X_{m}\left(B_{d}(t)\right)} \mid N\left(B_{d}(t)\right)=n\right\} \\
=\int_{-\infty}^{+\infty} \mathrm{e}^{\mathrm{i} \alpha x} \mathrm{P}\left\{X_{m}\left(B_{d}(t)\right) \in \mathrm{d} x \mid N\left(B_{d}(t)\right)=n\right\} \\
=\Gamma\left(v_{m}+1\right)\left(\frac{2}{\alpha c}\right)^{v_{m}} \frac{1}{\Gamma(d / 2) 2^{d / 2-1} t^{d / 2}} \int_{0}^{\infty} J_{v_{m}}(\alpha c s) s^{d-v_{m}-1} \mathrm{e}^{-s^{2} / 2 t} \mathrm{~d} s \\
=\frac{\Gamma\left(v_{m}+1\right)}{\Gamma(d / 2) 2^{d / 2-1} t^{d / 2}} \sum_{k=0}^{\infty} \frac{(-1)^{k}}{k ! \Gamma\left(k+v_{m}+1\right)}\left(\frac{\alpha c}{2}\right)^{2 k} \int_{0}^{\infty} s^{2 k+d-1} \mathrm{e}^{-s^{2} / 2 t} \mathrm{~d} s \\
=\frac{\Gamma\left(v_{m}+1\right)}{\Gamma(d / 2)} \sum_{k=0}^{\infty} \frac{(-1)^{k}}{k ! \Gamma\left(k+v_{m}+1\right)}\left(\frac{\alpha^{2} c^{2} t}{2}\right)^{k} \int_{0}^{\infty} y^{k+d / 2-1} \mathrm{e}^{-y} \mathrm{~d} y \\
=\frac{\Gamma\left(v_{m}+1\right)}{\Gamma(d / 2)} \sum_{k=0}^{\infty} \frac{(-1)^{k} \Gamma(k+d / 2)}{k ! \Gamma\left(k+v_{m}+1\right)}\left(\frac{\alpha^{2} c^{2} t}{2}\right)^{k} \\
=\frac{\Gamma\left(v_{m}+1\right)}{\Gamma(d / 2) \Gamma\left(v_{m}-d / 2+1\right)} \sum_{k=0}^{\infty} \frac{(-1)^{k}}{k !} B\left(k+\frac{d}{2}, v_{m}-\frac{d}{2}+1\right)\left(\frac{\alpha^{2} c^{2} t}{2}\right)^{k}
\end{aligned}
$$




$$
\begin{aligned}
& =\frac{1}{B\left(d / 2, v_{m}-d / 2+1\right)} \sum_{k=0}^{\infty} \frac{(-1)^{k}}{k !}\left(\frac{\alpha^{2} c^{2} t}{2}\right)^{k} \int_{0}^{1} w^{k+d / 2-1}(1-w)^{v_{m}-d / 2} \mathrm{~d} w \\
& =\frac{1}{B\left(d / 2, v_{m}-d / 2+1\right)} \int_{0}^{1} w^{d / 2-1}(1-w)^{v_{m}-d / 2} \mathrm{e}^{-\alpha^{2} c^{2} t w / 2} \mathrm{~d} w,
\end{aligned}
$$

where we have used the change of variable $y=s^{2} / 2 t$.

Finally, by inverting $\mathrm{E}\left\{\mathrm{e}^{\mathrm{i} \alpha X_{m}\left(B_{d}(t)\right)} \mid N\left(B_{d}(t)\right)=n\right\}$, result (3.2) emerges. This completes the proof.

The probability (3.2) claims that, conditionally on the number of events of $N\left(B_{d}(t)\right), t>0$, such that $v_{m}>d / 2-1$, the random process $X_{m}\left(B_{d}(t)\right), t>0$, is distributed as a centered Gaussian with variance $c^{2} t Z$, where $Z \sim B\left(d / 2, v_{m}-d / 2+1\right)$.

Remark 3.1. Under the condition that $v>d / 2-1$, by means of similar arguments exploited in the proof of Theorem 2.1, we obtain

$$
\begin{aligned}
& \mathrm{P}\left\{X_{v}\left(B_{d}(t)\right) \in \mathrm{d} x \mid N\left(B_{d}(t)\right)=0\right\} \\
& \quad=\frac{\mathrm{d} x}{B(d / 2, v-d / 2+1)} \int_{0}^{1} w^{d / 2-1}(1-w)^{\nu-d / 2} \frac{\mathrm{e}^{-x^{2} / 2 c^{2} t w}}{\sqrt{2 \pi t w} c} \mathrm{~d} w .
\end{aligned}
$$

In particular, we are interested in the random motions obtained by setting $v=0$ and $v=1$. It is clear that, for $v=0$, the condition $v>d / 2-1$ is satisfied for only $d=1$, whilst, for $d=2$, we have

$$
\mathrm{P}\left\{X_{0}\left(B_{2}(t)\right) \in \mathrm{d} x \mid N\left(B_{2}(t)\right)=0\right\}=\mathrm{d} x \frac{\mathrm{e}^{-x^{2} / 2 c^{2} t}}{\sqrt{2 \pi t} c} .
$$

If $v=1$, the above condition and representation (3.4) hold for both $d=1$ and $d=2$.

From (3.1) we can derive some well-known probability distributions. Indeed, for $d=1$, we obtain $B_{1}(t)=|W(t)|$, which is a reflected Brownian motion around the $x$-axis, and its density becomes $f_{1}(r)=\sqrt{2} \mathrm{e}^{-r^{2} / 2 t} / \sqrt{\pi t}$. Furthermore, $|W(t)|$ represents the Brownian time used in the definition of the iterated Brownian motion (see Allouba (2002)). For $d=2$, we obtain a Rayleigh random variable with $f_{2}(r)=r \mathrm{e}^{-r^{2} / 2 t} / t$, which also emerges by analyzing the distribution of the maximum of a Brownian bridge. Moreover, probability (3.2) holds for each $n \geq 1$, with the conditions $v_{m}>-\frac{1}{2}(d=1)$ and $v_{m}>0(d=2)$ always being satisfied.

In the next theorem we provide the unconditional probability distributions for $X_{m}(|W(t)|)$, $t>0$, and $X_{m}\left(B_{2}(t)\right), t>0$.

Theorem 3.2. For $d=1$, the following probability distribution holds:

$$
\mathrm{P}\left\{X_{m}(|W(t)|) \in \mathrm{d} x\right\}=\mathrm{d} x \int_{0}^{\infty} \mathrm{d} s f_{1}(s) \int_{-c s}^{c s} \frac{s \mathrm{e}^{-s^{2} x^{2} / 2 t y^{2}}}{\sqrt{2 \pi t y^{2}}} p^{m}(y, s) \mathrm{d} y, \quad x \in \mathbb{R}, m=0,1,
$$

where $p^{0}(y, t)$ and $p^{1}(y, t)$ are defined by (2.16) and (2.17), respectively. Furthermore, for 
$d=2$, we have

$$
\begin{aligned}
& \mathrm{P}\left\{X_{0}\left(B_{2}(t)\right) \in \mathrm{d} x\right\}= \mathrm{d} x \int_{0}^{\infty} \mathrm{d} s f_{2}(s) \mathrm{e}^{-\lambda s} \\
& \times\left(\frac{\lambda s}{2} \int_{0}^{1} \frac{\mathrm{e}^{\lambda s \sqrt{1-w}}}{\sqrt{1-w}} \frac{\mathrm{e}^{-x^{2} / 2 c^{2} t w}}{\sqrt{2 \pi t w} c} \mathrm{~d} w+\frac{\mathrm{e}^{-x^{2} / 2 c^{2} t}}{\sqrt{2 \pi t} c}\right), \\
& \mathrm{P}\left\{X_{1}\left(B_{2}(t)\right) \in \mathrm{d} x\right\}=\mathrm{d} x \int_{0}^{\infty} \mathrm{d} s f_{2}(s) \int_{0}^{1} \mathrm{e}^{-\lambda s w}[1+\lambda s(1-w)] \frac{\mathrm{e}^{-x^{2} / 2 c^{2} t w}}{\sqrt{2 \pi t w} c} \mathrm{~d} w .
\end{aligned}
$$

Proof. By taking into account (3.2) and (3.4), for $m=0\left(v_{0}=n / 2\right)$ and $d=1$, we obtain

$$
\begin{aligned}
& \frac{1}{\mathrm{~d} x} \mathrm{P}\left\{X_{0}(|W(t)|) \in \mathrm{d} x\right\} \\
& =\frac{1}{\mathrm{~d} x} \sum_{n=0}^{\infty} \mathrm{P}\{N(|W(t)|)=n\} \mathrm{P}\left\{X_{0}(|W(t)|) \in \mathrm{d} x \mid N(|W(t)|)=n\right\} \\
& =\int_{0}^{\infty} f_{1}(s) \mathrm{d} s \mathrm{e}^{-\lambda s} \sum_{n=0}^{\infty} \frac{(\lambda s)^{n}}{n !} \frac{1}{B(1 / 2,(n+1) / 2)} \\
& \times \int_{0}^{1} w^{-1 / 2}(1-w)^{(n-1) / 2} \frac{\mathrm{e}^{-x^{2} / 2 c^{2} t w}}{\sqrt{2 \pi t w c}} \mathrm{~d} w \\
& =\int_{0}^{\infty} f_{1}(s) \mathrm{d} s \frac{\mathrm{e}^{-\lambda s}}{\sqrt{\pi}} \\
& \times \int_{0}^{1} w^{-1 / 2}(1-w)^{-1 / 2} \frac{\mathrm{e}^{-x^{2} / 2 c^{2} t w}}{\sqrt{2 \pi t w} c} \sum_{n=0}^{\infty} \frac{(\lambda s)^{n}}{n !}(1-w)^{n / 2} \frac{\Gamma(n / 2+1)}{\Gamma((n+1) / 2)} \mathrm{d} w \\
& =\int_{0}^{\infty} f_{1}(s) \mathrm{d} s \mathrm{e}^{-\lambda s} \\
& \times \int_{0}^{1} w^{-1 / 2}(1-w)^{-1 / 2} \frac{\mathrm{e}^{-x^{2} / 2 c^{2} t w}}{\sqrt{2 \pi t w} c} \sum_{n=0}^{\infty} \frac{1}{\Gamma^{2}((n+1) / 2)}\left(\frac{\lambda s}{2} \sqrt{1-w}\right)^{n} \mathrm{~d} w \\
& =\int_{0}^{\infty} f_{1}(s) \mathrm{d} s \frac{\lambda s \mathrm{e}^{-\lambda s}}{2} \\
& \times \int_{0}^{1} w^{-1 / 2} \frac{\mathrm{e}^{-x^{2} / 2 c^{2} t w}}{\sqrt{2 \pi t w c}} \sum_{n=0}^{\infty} \frac{1}{\Gamma^{2}((n+1) / 2)}\left(\frac{\lambda s}{2} \sqrt{1-w}\right)^{n-1} \mathrm{~d} w \\
& =\int_{0}^{\infty} f_{1}(s) \mathrm{d} s \frac{\lambda \mathrm{e}^{-\lambda s}}{c} \\
& \times \int_{0}^{c s} \frac{s \mathrm{e}^{-s^{2} x^{2} / 2 t y^{2}}}{\sqrt{2 \pi t y^{2}}} \sum_{n=0}^{\infty} \frac{1}{\Gamma^{2}((n+1) / 2)}\left(\frac{\lambda}{2 c} \sqrt{c^{2} s^{2}-y^{2}}\right)^{n-1} \mathrm{~d} y \\
& =\int_{0}^{\infty} f_{1}(s) \mathrm{d} s \int_{-c s}^{c s} \frac{s \mathrm{e}^{-s^{2} x^{2} / 2 t y^{2}}}{\sqrt{2 \pi t y^{2}}} \frac{\lambda \mathrm{e}^{-\lambda s}}{2 c} \sum_{n=0}^{\infty} \frac{1}{\Gamma^{2}((n+1) / 2)}\left(\frac{\lambda}{2 c} \sqrt{c^{2} s^{2}-y^{2}}\right)^{n-1} \mathrm{~d} y \text {, }
\end{aligned}
$$

where we have used the change of variable $y=c s \sqrt{w}$. 
For $m=1\left(v_{1}=n+1\right)$ and $d=1$, we have

$$
\begin{aligned}
\frac{1}{\mathrm{~d} x} \mathrm{P}\{ & \left.X_{1}(|W(t)|) \in \mathrm{d} x\right\} \\
= & \int_{0}^{\infty} f_{1}(s) \mathrm{d} s \mathrm{e}^{-\lambda s} \sum_{n=0}^{\infty} \frac{(\lambda s)^{n}}{n !} \frac{1}{B(1 / 2, n+3 / 2)} \int_{0}^{1} w^{-1 / 2}(1-w)^{n+1 / 2} \frac{\mathrm{e}^{-x^{2} / 2 c^{2} t w}}{\sqrt{2 \pi t w} c} \mathrm{~d} w \\
= & \int_{0}^{\infty} f_{1}(s) \mathrm{d} s \frac{\mathrm{e}^{-\lambda s}}{\sqrt{\pi}} \int_{0}^{1} w^{-1 / 2} \frac{\mathrm{e}^{-x^{2} / 2 c^{2} t w}}{\sqrt{2 \pi t w} c} \sum_{n=0}^{\infty} \frac{(\lambda s)^{n}}{n !}(1-w)^{n+1 / 2} \frac{\Gamma(n+2)}{\Gamma(n+3 / 2)} \mathrm{d} w \\
= & \int_{0}^{\infty} f_{1}(s) \mathrm{d} s \frac{\mathrm{e}^{-\lambda s}}{\sqrt{\pi \lambda s}} \int_{0}^{1} w^{-1 / 2} \frac{\mathrm{e}^{-x^{2} / 2 c^{2} t w}}{\sqrt{2 \pi t w} c} \sum_{n=0}^{\infty} \frac{n+1}{\Gamma(n+3 / 2)}(\lambda s(1-w))^{n+1 / 2} \mathrm{~d} w \\
= & \int_{0}^{\infty} f_{1}(s) \mathrm{d} s \frac{2}{c} \frac{\mathrm{e}^{-\lambda s}}{\sqrt{\lambda \pi s^{3}}} \\
& \times \int_{0}^{c s} \frac{s \mathrm{e}^{-s^{2} x^{2} / 2 t y^{2}}}{\sqrt{2 \pi t y^{2}}} \sum_{n=0}^{\infty} \frac{n+1}{\Gamma(n+3 / 2)}\left(\frac{\lambda}{c^{2} s}\left(c^{2} s^{2}-y^{2}\right)\right)^{n+1 / 2} \mathrm{~d} y \\
= & \int_{0}^{\infty} f_{1}(s) \mathrm{d} s \\
& \times \int_{-c s}^{c s} \frac{s \mathrm{e}^{-s^{2} x^{2} / 2 t y^{2}}}{\sqrt{2 \pi t y^{2}}} \frac{\mathrm{e}^{-\lambda s}}{c \sqrt{\lambda \pi s^{3}}} \sum_{n=0}^{\infty} \frac{n+1}{\Gamma(n+3 / 2)}\left(\frac{\lambda}{c^{2} s}\left(c^{2} s^{2}-y^{2}\right)\right)^{n+1 / 2} \mathrm{~d} y,
\end{aligned}
$$

where we have used the change of variable $y=c s \sqrt{w}$. Therefore, result (3.5) is proved. Developing the quantity

$$
\int_{0}^{\infty} f_{2}(s) \mathrm{d} s \mathrm{e}^{-\lambda s} \sum_{n=0}^{\infty} \frac{(\lambda s)^{n}}{n !} \mathrm{P}\left\{X_{m}\left(B_{2}(t)\right) \in \mathrm{d} x \mid N\left(B_{2}(t)\right)=n\right\}
$$

for $m=0,1$, and taking into account Remark 3.1, it is not hard to prove results (3.6) and (3.7).

The probability distribution (3.5) permits us to claim that the process $X_{m}(t)$ stopped at a reflected Brownian time is equivalent in distribution to a Brownian motion with variance $t X_{m}^{2}(|W(t)|) / W^{2}(t)$.

Remark 3.2. Recalling that, for a centered Gaussian with variance $\sigma^{2}$, the moments are given by

$$
\sigma^{p} \frac{\Gamma(p+1)}{2^{p / 2} \Gamma(p / 2+1)},
$$

if $p$ is even, and are 0 if $p$ is odd, we obtain

$$
\mathrm{E}\left\{X_{m}^{p}(|W(t)|)\right\}=\frac{t^{p / 2} \Gamma(p+1)}{2^{p / 2} \Gamma(p / 2+1)} \int_{0}^{\infty} \frac{f_{1}(s)}{s^{p}} \mathrm{E}\left\{X_{m}^{p}(s)\right\} \mathrm{d} s,
$$

where $\mathrm{E}\left\{X_{m}^{p}(t)\right\}$ is defined as in Theorem 2.5 and $p$ is even.

Now, we analyze the effect of the random clock defined as the time spent on the positive axis (sojourn time) by a standard Brownian motion $W(t), t>0$, namely,

$$
\Gamma(t)=\int_{0}^{t} \mathbf{1}_{\{W(s)>0\}}(s) \mathrm{d} s .
$$


The density function of $\Gamma(t)$ is $\gamma(s)=1 / \pi \sqrt{s(t-s)}, 0<s<t$, also known as the arcsin law. We have the following result concerning $X_{m}(\Gamma(t)), t>0$, where $\Gamma(t)$ and $X_{m}(t)$ are considered to be independent.

Theorem 3.3. For $n \geq 1$, the following probability distribution holds:

$$
\begin{aligned}
& \mathrm{P}\left\{X_{m}(\Gamma(t)) \in \mathrm{d} x \mid N(\Gamma(t))=n\right\} \\
& \quad=\frac{\mathrm{d} x}{B\left(1, v_{m}\right)} \int_{0}^{t} \gamma(s) \mathrm{d} s \int_{0}^{1}(1-w)^{v_{m}-1} \mathrm{~d} w \frac{1}{2 \pi} \frac{1}{\sqrt{c^{2} s^{2} w-x^{2}}}
\end{aligned}
$$

with $m=0,1$.

Proof. Applying the same arguments as those used to prove (3.3), we obtain

$$
\begin{aligned}
& \mathrm{P}\left\{X_{m}(\Gamma(t)) \in \mathrm{d} x \mid N(\Gamma(t))=n\right\} \\
& \quad=\frac{\mathrm{d} x}{2 \pi} \frac{\Gamma\left(v_{m}+1\right) \Gamma\left(v_{m}\right)}{\Gamma\left(2 v_{m}\right)} \int_{0}^{t}\left(\frac{2}{c s}\right)^{v_{m}}\left(c^{2} s^{2}-x^{2}\right)^{v_{m}-1 / 2} \mathbf{1}_{\{|x|<c s\}} \gamma(s) \mathrm{d} s .
\end{aligned}
$$

As before, we consider the Fourier transform for $X_{m}(\Gamma(t)), t>0$. Hence, we obtain

$$
\begin{aligned}
& \mathrm{E}\left\{\mathrm{e}^{\mathrm{i} \alpha X_{m}(\Gamma(t))} \mid N(\Gamma(t))=n\right\} \\
& =\frac{\Gamma\left(v_{m}+1\right)}{\pi} \sum_{k=0}^{\infty} \frac{(-1)^{k}}{k ! \Gamma\left(k+v_{m}+1\right)}\left(\frac{\alpha c}{2}\right)^{2 k} \int_{0}^{t} s^{2 k-1 / 2}(t-s)^{-1 / 2} \mathrm{~d} s \\
& =\frac{\Gamma\left(v_{m}+1\right)}{\pi} \sum_{k=0}^{\infty} \frac{(-1)^{k}}{k ! \Gamma\left(k+v_{m}+1\right)}\left(\frac{\alpha c t}{2}\right)^{2 k} \int_{0}^{1} y^{2 k-1 / 2}(1-y)^{-1 / 2} \mathrm{~d} y \\
& =\frac{\Gamma\left(v_{m}+1\right)}{\pi} \sum_{k=0}^{\infty} \frac{(-1)^{k} \Gamma(2 k+1 / 2) \Gamma(1 / 2)}{k ! \Gamma\left(k+v_{m}+1\right) \Gamma(2 k+1)}\left(\frac{\alpha c t}{2}\right)^{2 k} \\
& =\frac{v_{m}}{\pi} \sum_{k=0}^{\infty} \frac{(-1)^{k} \Gamma(2 k+1 / 2) \Gamma(k+1) \Gamma\left(v_{m}\right) \Gamma(1 / 2)}{k ! \Gamma(k+1) \Gamma\left(k+v_{m}+1\right) \Gamma(2 k+1)}\left(\frac{\alpha c t}{2}\right)^{2 k} \\
& =\frac{v_{m}}{\pi} \sum_{k=0}^{\infty} \frac{(-1)^{k}}{(k !)^{2}} B\left(2 k+\frac{1}{2}, \frac{1}{2}\right) B\left(k+1, v_{m}\right)\left(\frac{\alpha c t}{2}\right)^{2 k} \\
& =\frac{v_{m}}{\pi} \int_{0}^{1} z^{-1 / 2}(1-z)^{-1 / 2} \mathrm{~d} z \int_{0}^{1}(1-w)^{v_{m}-1} \mathrm{~d} w J_{0}(\alpha c t z \sqrt{w}),
\end{aligned}
$$

where we have used the change of variable $s=t y$. Therefore, by taking into account Theorems 2.1-2.4, we obtain

$$
\begin{aligned}
\frac{1}{\mathrm{~d} x} \mathrm{P} & \left\{X_{m}(\Gamma(t)) \in \mathrm{d} x \mid N(\Gamma(t))=n\right\} \\
& =\frac{v_{m}}{\pi} \int_{0}^{1} z^{-1 / 2}(1-z)^{-1 / 2} \mathrm{~d} z \int_{0}^{1}(1-w)^{v_{m}-1} \mathrm{~d} w \frac{1}{2 \pi} \frac{1}{\sqrt{c^{2} t^{2} z^{2} w-x^{2}}} \\
& =\frac{v_{m}}{\pi} \int_{0}^{t} \frac{\mathrm{d} s}{\sqrt{s(t-s)}} \int_{0}^{1}(1-w)^{v_{m}-1} \mathrm{~d} w \frac{1}{2 \pi} \frac{1}{\sqrt{c^{2} s^{2} w-x^{2}}}
\end{aligned}
$$

where we have used the change of variable $s=t z$. This completes the proof. 
From Theorem 3.3 we conclude that $X_{m}(t), t>0$, with random time $\Gamma(t), t>0$, is equivalent in distribution to the random motion $X_{0}(t), t>0$, with a random time given by $\Gamma(t) \sqrt{Z}$, where $Z \sim B\left(1, v_{m}\right)$, which maintains the velocity initially chosen until $t$.

Remark 3.3. The random process $\Gamma(t), t>0$, is also connected with $X_{m}\left(B_{d}(t)\right), t>0$. Indeed, recalling that

$$
\mathrm{P}\{\Gamma(t) \in \mathrm{d} s \mid W(t)>0\}=\frac{2}{\pi t} \frac{\sqrt{s}}{\sqrt{(t-s)}} \mathrm{d} s, \quad \mathrm{P}\{\Gamma(t) \in \mathrm{d} s \mid W(t)<0\}=\frac{2}{\pi t} \frac{\sqrt{t-s}}{\sqrt{s}} \mathrm{~d} s
$$

for $0<s<t$, from (3.2) and (3.4), the following equalities hold:

$$
\begin{aligned}
\mathrm{P}\left\{X_{0}(|W(t)|) \in \mathrm{d} x \mid N(|W(t)|)=0\right\} & =\mathrm{P}\left\{\tilde{W}\left(c^{2} \Gamma(t)\right) \in \mathrm{d} x\right\} \\
& =\frac{\mathrm{d} x}{\pi} \int_{0}^{t} \frac{1}{\sqrt{s(t-s)}} \frac{\mathrm{e}^{-x^{2} / 2 c^{2} s}}{\sqrt{2 \pi s} c} \mathrm{~d} s, \\
\mathrm{P}\left\{X_{0}\left(B_{3}(t)\right) \in \mathrm{d} x \mid N\left(B_{3}(t)\right)=2\right\} & =\mathrm{P}\left\{X_{1}\left(B_{3}(t)\right) \in \mathrm{d} x \mid N\left(B_{3}(t)\right)=0\right\} \\
& =\mathrm{P}\left\{\tilde{W}\left(c^{2} \Gamma(t)\right) \in \mathrm{d} x \mid W(t)>0\right\} \\
& =\frac{\mathrm{d} x 2}{\pi t} \int_{0}^{t} \frac{\sqrt{s}}{\sqrt{(t-s)}} \frac{\mathrm{e}^{-x^{2} / 2 c^{2} s}}{\sqrt{2 \pi s} c} \mathrm{~d} s, \\
\mathrm{P}\left\{X_{0}(|W(t)|) \in \mathrm{d} x \mid N(|W(t)|)=2\right\} & =\mathrm{P}\left\{X_{1}(|W(t)|) \in \mathrm{d} x \mid N(|W(t)|)=0\right\} \\
& =\mathrm{P}\left\{\tilde{W}\left(c^{2} \Gamma(t)\right) \in \mathrm{d} x \mid W(t)<0\right\} \\
& =\frac{\mathrm{d} x 2}{\pi t} \int_{0}^{t} \frac{\sqrt{t-s}}{\sqrt{s}} \frac{\mathrm{e}^{-x^{2} / 2 c^{2} s}}{\sqrt{2 \pi s} c} \mathrm{~d} s,
\end{aligned}
$$

where $\tilde{W}(t)$ is an independent Brownian motion with respect to $W(t)$.

\subsection{Compositions with gamma random times}

In this subsection of the paper we deal with a second class of random times, different from the previous class. Let $G_{\alpha}(t), t>0$, a gamma random process, with parameter $\alpha>0$, be governed by the density law $g_{\alpha}(s, t)=t^{\alpha} s^{\alpha-1} \mathrm{e}^{-t s} / \Gamma(\alpha), s>0$. The process $N\left(G_{\alpha}(t)\right), t>0$, governs the changes in velocity of $X_{m}\left(G_{\alpha}(t)\right), t>0\left(G_{\alpha}(t), t>0\right.$, and $X_{m}(t), t>0$, are assumed to be independent), and has distribution given by

$$
\mathrm{P}\left\{N\left(G_{\alpha}(t)\right)=n\right\}=\int_{0}^{\infty} \mathrm{P}\{N(s)=n\} g_{\alpha}(s, t) \mathrm{d} s, \quad n \geq 0 .
$$

Analogously to the Bessel case, we study the conditional probability distribution of the random motion $X_{m}\left(G_{\alpha}(t)\right), t>0$.

Theorem 3.4. Given $N\left(G_{\alpha}(t)\right)=n \geq 1$, such that $v_{m}>\alpha / 2-1$, the random process $X_{m}\left(G_{\alpha}(t)\right)$ has the following conditional probability distribution:

$$
\begin{aligned}
\mathrm{P}\left\{X_{m}\left(G_{\alpha}(t)\right) \in \mathrm{d} x \mid N\left(G_{\alpha}(t)\right)=n\right\} \\
=\frac{\mathrm{d} x}{\Gamma((\alpha+1) / 2) B\left(\alpha / 2, v_{m}-\alpha / 2+1\right)} \\
\quad \times \int_{0}^{1} \mathrm{~d} w w^{\alpha / 2-1}(1-w)^{v_{m}-\alpha / 2} \frac{t}{\sqrt{\pi w} c}\left(\frac{t|x|}{2 c \sqrt{w}}\right)^{\alpha / 2} K_{-\alpha / 2}\left(\frac{t|x|}{c \sqrt{w}}\right)
\end{aligned}
$$

where $K_{\mu}(x)$ is the modified Bessel function of the second kind and $m=0,1$. 
Proof. The random process $X_{m}\left(G_{\alpha}(t)\right), t>0$, admits as support the real line; hence, the following probability distribution holds:

$$
\begin{aligned}
\mathrm{P}\left\{X_{m}\right. & \left.\left(G_{\alpha}(t)\right) \in \mathrm{d} x \mid N\left(G_{\alpha}(t)\right)=n\right\} \\
= & \int_{0}^{\infty} \mathrm{P}\left\{X_{m}\left(G_{\alpha}(t)\right) \in \mathrm{d} x \mid N\left(G_{\alpha}(t)\right)=n, G_{\alpha}(t)=s\right\} \mathrm{P}\left\{G_{\alpha}(t) \in \mathrm{d} s\right\} \\
= & \int_{0}^{\infty} \mathrm{P}\left\{X_{m}\left(G_{\alpha}(t)\right) \in \mathrm{d} x \mid N(s)=n\right\} \mathrm{P}\left\{G_{\alpha}(t) \in \mathrm{d} s\right\} \\
= & \frac{\mathrm{d} x}{2 \pi} \frac{\Gamma\left(v_{m}+1\right) \Gamma\left(v_{m}\right)}{\Gamma\left(2 v_{m}\right)} \frac{t^{\alpha}}{\Gamma(\alpha)} \\
& \times \int_{0}^{\infty}\left(\frac{2}{c s}\right)^{v_{m}}\left(c^{2} s^{2}-x^{2}\right)^{v_{m}-1 / 2} \mathbf{1}_{\{|x|<c s\}} s^{\alpha-1} \mathrm{e}^{-t s} \mathrm{~d} s .
\end{aligned}
$$

Then

$$
\begin{aligned}
& \mathrm{E}\left\{\mathrm{e}^{\mathrm{i} \alpha X_{m}\left(G_{\alpha}(t)\right)} \mid N\left(G_{\alpha}(t)\right)=n\right\} \\
& =\Gamma\left(v_{m}+1\right)\left(\frac{2}{\alpha c}\right)^{v_{m}} \frac{t^{\alpha}}{\Gamma(\alpha)} \int_{0}^{\infty} \frac{J_{v_{m}}(\alpha c s)}{s^{v_{m}}} s^{\alpha-1} \mathrm{e}^{-t s} \mathrm{~d} s \\
& =\Gamma\left(v_{m}+1\right) \frac{t^{\alpha}}{\Gamma(\alpha)} \sum_{k=0}^{\infty} \frac{(-1)^{k}}{k ! \Gamma\left(k+v_{m}+1\right)}\left(\frac{\alpha c}{2}\right)^{2 k} \int_{0}^{\infty} s^{2 k+\alpha-1} \mathrm{e}^{-t s} \mathrm{~d} s \\
& =\frac{\Gamma\left(v_{m}+1\right)}{\Gamma(\alpha)} \sum_{k=0}^{\infty} \frac{(-1)^{k} \Gamma(2 k+\alpha)}{k ! \Gamma\left(k+v_{m}+1\right)}\left(\frac{\alpha c}{2 t}\right)^{2 k} \\
& =\frac{\Gamma\left(v_{m}+1\right)}{\sqrt{\pi} \Gamma(\alpha)} \sum_{k=0}^{\infty} \frac{(-1)^{k} \Gamma(k+\alpha / 2) \Gamma(k+(\alpha+1) / 2) 2^{2 k+\alpha-1}}{k ! \Gamma\left(k+v_{m}+1\right)}\left(\frac{\alpha c}{2 t}\right)^{2 k} \\
& =\frac{\Gamma\left(v_{m}+1\right) 2^{\alpha-1}}{\sqrt{\pi} \Gamma(\alpha) \Gamma\left(v_{m}-\alpha / 2+1\right)} \\
& \times \sum_{k=0}^{\infty} \frac{(-1)^{k}}{k !}\left(\frac{\alpha c}{t}\right)^{2 k} \Gamma\left(k+\frac{\alpha+1}{2}\right) B\left(k+\frac{\alpha}{2}, v_{m}-\frac{\alpha}{2}+1\right) \\
& =\frac{\Gamma\left(v_{m}+1\right)}{\Gamma(\alpha / 2) \Gamma((\alpha+1) / 2) \Gamma\left(v_{m}-\alpha / 2+1\right)} \\
& \times \sum_{k=0}^{\infty} \frac{(-1)^{k}}{k !}\left(\frac{\alpha c}{t}\right)^{2 k} \Gamma\left(k+\frac{\alpha+1}{2}\right) B\left(k+\frac{\alpha}{2}, v_{m}-\frac{\alpha}{2}+1\right) \\
& =\frac{1}{\Gamma((\alpha+1) / 2) B\left(\alpha / 2, v_{m}-\alpha / 2+1\right)} \\
& \times \sum_{k=0}^{\infty} \frac{(-1)^{k}}{k !}\left(\frac{\alpha c}{t}\right)^{2 k} \int_{0}^{\infty} \mathrm{e}^{-z} z^{k+(\alpha+1) / 2-1} \mathrm{~d} z \int_{0}^{1} w^{k+\alpha / 2-1}(1-w)^{v_{m}-\alpha / 2} \mathrm{~d} w \\
& =\frac{1}{\Gamma((\alpha+1) / 2) B\left(\alpha / 2, v_{m}-\alpha / 2+1\right)} \int_{0}^{\infty} \mathrm{e}^{-z} z^{(\alpha+1) / 2-1} \mathrm{~d} z \\
& \times \int_{0}^{1} w^{\alpha / 2-1}(1-w)^{v_{m}-\alpha / 2} \mathrm{~d} w \mathrm{e}^{-(\alpha c / t)^{2} z w}
\end{aligned}
$$


By inverting the above quantity, we obtain

$$
\begin{aligned}
\frac{1}{\mathrm{~d} x} \mathrm{P}\{ & \left.X_{m}\left(G_{\alpha}(t)\right) \in \mathrm{d} x \mid N\left(G_{\alpha}(t)\right)=n\right\} \\
= & \frac{1}{\Gamma((\alpha+1) / 2)} \int_{0}^{\infty} \mathrm{d} z \mathrm{e}^{-z} z^{(\alpha+1) / 2-1} \frac{1}{B\left(\alpha / 2, v_{m}-\alpha / 2+1\right)} \\
& \times \int_{0}^{1} \mathrm{~d} w w^{\alpha / 2-1}(1-w)^{v_{m}-\alpha / 2} \frac{t \mathrm{e}^{-t^{2} x^{2} / 4 c^{2} z w}}{\sqrt{4 \pi z w} c} \\
= & \frac{1}{\Gamma((\alpha+1) / 2)} \frac{1}{B\left(\alpha / 2, v_{m}-\alpha / 2+1\right)} \\
& \times \int_{0}^{1} \mathrm{~d} w w^{\alpha / 2-1}(1-w)^{v_{m}-\alpha / 2} \frac{t}{\sqrt{\pi w} c}\left(\frac{t|x|}{2 c \sqrt{w}}\right)^{\alpha / 2} K_{-\alpha / 2}\left(\frac{t|x|}{c \sqrt{w}}\right),
\end{aligned}
$$

where in the last step we used the following integral representation for the modified Bessel functions $K_{\mu}(x)$ :

$$
K_{\mu}(x)=\frac{1}{2}\left(\frac{x}{2}\right)^{\mu} \int_{0}^{\infty} \mathrm{e}^{-z-x^{2} / 4 z} z^{-\mu-1} \mathrm{~d} z .
$$

Remark 3.4. From (3.9), it is straightforward to observe that

$$
\mathrm{P}\left\{X_{m}\left(G_{\alpha}(t)\right) \in \mathrm{d} x \mid N\left(G_{\alpha}(t)\right)=n\right\}
$$

coincides with the density of a Gaussian random variable with variance $2 c^{2} U Z / t^{2}$, where $U$ is a gamma variable with parameters $(\alpha+1) / 2$ and 1 , while $Z \sim B\left(\alpha / 2, v_{m}-\alpha / 2+1\right)$.

Remark 3.5. The approach developed in the previous proof is sufficient to show that

$$
\begin{aligned}
\mathrm{P}\left\{X_{v}\left(G_{\alpha}(t)\right) \in \mathrm{d} x \mid N\left(G_{\alpha}(t)\right)=0\right\} \\
=\frac{\mathrm{d} x}{\Gamma((\alpha+1) / 2) B(\alpha / 2, v-\alpha / 2+1)} \\
\quad \times \int_{0}^{1} \mathrm{~d} w w^{\alpha / 2-1}(1-w)^{\nu-\alpha / 2} \frac{t}{\sqrt{\pi w} c}\left(\frac{t|x|}{2 c \sqrt{w}}\right)^{\alpha / 2} K_{-\alpha / 2}\left(\frac{t|x|}{c \sqrt{w}}\right)
\end{aligned}
$$

with $v>\alpha / 2-1$.

For $\alpha=1, G_{1}(t), t>0$, becomes an exponential random process. The exponential clock permits us to derive an interesting interpretation of probability (3.8). Indeed, since

$$
K_{ \pm 1 / 2}(x)=\sqrt{\frac{\pi}{2 x}} \mathrm{e}^{-x}
$$

we obtain

$$
\begin{aligned}
& \mathrm{P}\left\{X_{m}\left(G_{1}(t)\right) \in \mathrm{d} x \mid N\left(G_{1}(t)\right)=n\right\} \\
& \quad=\frac{1}{B\left(1 / 2, v_{m}+1 / 2\right)} \int_{0}^{1} \mathrm{~d} w w^{-1 / 2}(1-w)^{v_{m}-1 / 2} \frac{t}{2 c \sqrt{w}} \mathrm{e}^{-t|x| / c \sqrt{w}}
\end{aligned}
$$

for $n \geq 0$, with the condition $v_{m}>-\frac{1}{2}$ always being satisfied. This means that the conditional probability of $X_{m}\left(G_{1}(t)\right), t>0$, is equivalent to the distribution of a Laplace random variable with parameter $t / c \sqrt{Z}$, where $Z \sim B\left(\frac{1}{2}, v_{m}+\frac{1}{2}\right)$. 
Remark 3.6. The process $X_{0}\left(G_{1}(t)\right), t>0$, and the occupation time $\Gamma(t), t>0$, are linked by the relationships

$$
\begin{aligned}
\mathrm{P}\left\{X_{0}\left(G_{1}(t)\right) \in \mathrm{d} x \mid N\left(G_{1}(t)\right)=0\right\} & =\mathrm{d} x \int_{0}^{t} \mathrm{P}\{\Gamma(t) \in \mathrm{d} s\} \frac{t^{3 / 2}}{2 c \sqrt{s}} \mathrm{e}^{-t^{3 / 2}|x| / c \sqrt{s}}, \\
\mathrm{P}\left\{X_{0}\left(G_{1}(t)\right) \in \mathrm{d} x \mid N\left(G_{1}(t)\right)=2\right\} & =\mathrm{P}\left\{X_{1}\left(G_{1}(t)\right) \in \mathrm{d} x \mid N\left(G_{1}(t)\right)=0\right\} \\
& =\mathrm{d} x \int_{0}^{t} \mathrm{P}\{\Gamma(t) \in \mathrm{d} s \mid W(t)<0\} \frac{t^{3 / 2}}{2 c \sqrt{s}} \mathrm{e}^{-t^{3 / 2}|x| / c \sqrt{s}},
\end{aligned}
$$

which follow from (3.10) and (3.11).

Now, by means of the same steps used in the proof of Theorem 3.2, it is not difficult to prove the result contained in the next theorem. Therefore, we omit the proof.

Theorem 3.5. For $\alpha=1$, we have

$$
\mathrm{P}\left\{X_{m}\left(G_{1}(t)\right) \in \mathrm{d} x\right\}=\mathrm{d} x \int_{0}^{\infty} g_{1}(s, t) \mathrm{d} s \int_{0}^{c s} \frac{s t}{2 y} \mathrm{e}^{-s t|x| / y} q^{m}(y, s) \mathrm{d} y,
$$

where $q^{m}(y, t)=2 p^{m}(y, t), m=0,1$, and $p^{0}(y, t)$ and $p^{1}(y, t)$ are respectively defined by (2.16) and (2.17).

Theorem 3.5 claims that $X_{m}(t)$ with exponential time is equivalent in distribution to a Laplace random variable with parameter $\left|X_{m}\left(G_{1}(t)\right)\right| / t G_{1}(t)$.

In order to conclude the discussion on random times, we observe that $B_{d}(t)$ and $G_{\alpha}(t)$ can be composed to obtain a new class of random times. Obviously, $B_{d}(t)$ and $G_{\alpha}(t)$ are thought to be mutually independent.

Theorem 3.6. For $n \geq 1$ and $v_{m}>d / 2-1$, we have

$$
\begin{aligned}
\mathrm{P}\left\{X_{m}\right. & \left.\left(B_{d}\left(G_{\alpha}(t)\right)\right) \in \mathrm{d} x \mid N\left(B_{d}\left(G_{\alpha}(t)\right)\right)=n\right\} \\
= & \frac{\mathrm{d} x}{\Gamma(\alpha) B\left(d / 2, v_{m}-d / 2+1\right)} \\
& \times \int_{0}^{1} \mathrm{~d} w w^{d / 2-1}(1-w)^{v_{m}-d / 2} \frac{\sqrt{2 t}}{\sqrt{\pi w} c}\left(\frac{\sqrt{t}|x|}{c \sqrt{2 w}}\right)^{\alpha-1 / 2} K_{-\alpha+1 / 2}\left(\frac{\sqrt{2 t}|x|}{c \sqrt{w}}\right) .
\end{aligned}
$$

Proof. Since $B_{d}\left(G_{\alpha}(t)\right)$ has a density given by

$$
\frac{t^{\alpha}}{\Gamma(\alpha) \Gamma(d / 2) 2^{d / 2-1}} \int_{0}^{\infty} \frac{r^{d-1}}{z^{d / 2}} \mathrm{e}^{-r^{2} / 2 z} z^{\alpha-1} \mathrm{e}^{-t z} \mathrm{~d} z
$$

we can write

$$
\begin{aligned}
\mathrm{P}\left\{X_{m}\left(B_{d}\left(G_{\alpha}(t)\right)\right) \in \mathrm{d} x \mid N\left(B_{d}\left(G_{\alpha}(t)\right)\right)=n\right\} \\
=\frac{\Gamma\left(v_{m}+1\right) \Gamma\left(v_{m}\right)}{2 \pi \Gamma\left(2 v_{m}\right)} \frac{t^{\alpha} \mathrm{d} x}{\Gamma(\alpha) \Gamma(d / 2) 2^{d / 2-1}} \\
\quad \times \int_{0}^{\infty} \mathrm{d} s \int_{0}^{\infty} \mathrm{d} z\left(\frac{2}{c s}\right)^{v_{m}}\left(c^{2} s^{2}-x^{2}\right)^{v_{m}-1 / 2} \mathbf{1}_{\{|x|<c s\}} \frac{s^{d-1}}{z^{d / 2}} \mathrm{e}^{-s^{2} / 2 z} z^{\alpha-1} \mathrm{e}^{-t z} .
\end{aligned}
$$


Therefore, by means of Theorem 2.3, we are able to explicit the Fourier transform of the previous probability distribution as follows:

$$
\begin{aligned}
& \int_{-\infty}^{+\infty} \mathrm{e}^{\mathrm{i} \alpha x} \mathrm{P}\left\{X_{m}\left(B_{d}\left(G_{\alpha}(t)\right)\right) \in \mathrm{d} x \mid N\left(B_{d}\left(G_{\alpha}(t)\right)\right)=n\right\} \\
& =\Gamma\left(v_{m}+1\right)\left(\frac{2}{\alpha c}\right)^{v_{m}} \frac{t^{\alpha}}{\Gamma(\alpha) \Gamma(d / 2) 2^{d / 2-1}} \int_{0}^{\infty} z^{\alpha-d / 2-1} \mathrm{e}^{-t z} \mathrm{~d} z \\
& \times \int_{0}^{\infty} J_{v_{m}}(\alpha c s) s^{d-v_{m}-1} \mathrm{e}^{-s^{2} / 2 z} \mathrm{~d} s \\
& =\frac{t^{\alpha} \Gamma\left(v_{m}+1\right)}{\Gamma(\alpha) \Gamma(d / 2) 2^{d / 2-1}} \int_{0}^{\infty} z^{\alpha-d / 2-1} \mathrm{e}^{-t z} \mathrm{~d} z \\
& \times \sum_{k=0}^{\infty} \frac{(-1)^{k}}{k ! \Gamma\left(k+v_{m}+1\right)}\left(\frac{\alpha c}{2}\right)^{2 k} \int_{0}^{\infty} s^{2 k+d-1} \mathrm{e}^{-s^{2} / 2 z} \mathrm{~d} s \\
& =\frac{t^{\alpha} \Gamma\left(v_{m}+1\right)}{\Gamma(\alpha) \Gamma(d / 2)} \int_{0}^{\infty} z^{\alpha-1} \mathrm{e}^{-t z} \mathrm{~d} z \\
& \times \sum_{k=0}^{\infty} \frac{(-1)^{k}}{k ! \Gamma\left(k+v_{m}+1\right)}\left(\frac{\alpha^{2} c^{2} z}{2}\right)^{k} \int_{0}^{\infty} y^{k+d / 2-1} \mathrm{e}^{-y} \mathrm{~d} y \\
& =\frac{t^{\alpha} \Gamma\left(v_{m}+1\right)}{\Gamma(\alpha) \Gamma(d / 2)} \int_{0}^{\infty} z^{\alpha-1} \mathrm{e}^{-t z} \mathrm{~d} z \sum_{k=0}^{\infty} \frac{(-1)^{k} \Gamma(k+d / 2)}{k ! \Gamma\left(k+v_{m}+1\right)}\left(\frac{\alpha^{2} c^{2} z}{2}\right)^{k} \\
& =\frac{t^{\alpha} \Gamma\left(v_{m}+1\right)}{\Gamma(\alpha) \Gamma(d / 2) \Gamma\left(v_{m}-d / 2+1\right)} \int_{0}^{\infty} z^{\alpha-1} \mathrm{e}^{-t z} \mathrm{~d} z \\
& \times \sum_{k=0}^{\infty} \frac{(-1)^{k}}{k !} B\left(k+\frac{d}{2}, v_{m}-\frac{d}{2}+1\right)\left(\frac{\alpha^{2} c^{2} z}{2}\right)^{k} \\
& =\frac{t^{\alpha}}{\Gamma(\alpha) B\left(d / 2, v_{m}-d / 2+1\right)} \int_{0}^{\infty} z^{\alpha-1} \mathrm{e}^{-t z} \mathrm{~d} z \\
& \times \sum_{k=0}^{\infty} \frac{(-1)^{k}}{k !}\left(\frac{\alpha^{2} c^{2} z}{2}\right)^{k} \int_{0}^{1} w^{k+d / 2-1}(1-w)^{v_{m}-d / 2} \mathrm{~d} w \\
& =\frac{t^{\alpha}}{\Gamma(\alpha) B\left(d / 2, v_{m}-d / 2+1\right)} \int_{0}^{\infty} z^{\alpha-1} \mathrm{e}^{-t z} \mathrm{~d} z \\
& \times \int_{0}^{1} w^{d / 2-1}(1-w)^{v_{m}-d / 2} \mathrm{e}^{-\alpha^{2} c^{2} z w / 2} \mathrm{~d} w,
\end{aligned}
$$

where we have used the change of variable $y=s^{2} / 2 z$.

By inverting the above characteristic function, we obtain

$$
\begin{aligned}
\mathrm{P}\left\{X_{m}(\right. & \left.\left.B_{d}\left(G_{\alpha}(t)\right)\right) \in \mathrm{d} x \mid N\left(B_{d}\left(G_{\alpha}(t)\right)\right)=n\right\} \\
= & \frac{t^{\alpha}}{\Gamma(\alpha) B\left(d / 2, v_{m}-d / 2+1\right)} \int_{0}^{1} w^{d / 2-1}(1-w)^{v_{m}-d / 2} \mathrm{~d} w \\
& \times \int_{0}^{\infty} z^{\alpha-1} \frac{\mathrm{e}^{-t z-x^{2} / 2 c^{2} z w}}{\sqrt{2 \pi z w} c} \mathrm{~d} z
\end{aligned}
$$




$$
\begin{aligned}
= & \frac{1}{\Gamma(\alpha) B\left(d / 2, v_{m}-d / 2+1\right)} \int_{0}^{1} w^{d / 2-1}(1-w)^{v_{m}-d / 2} \mathrm{~d} w \\
& \times \int_{0}^{\infty} u^{\alpha-1 / 2-1} \frac{\sqrt{t} \mathrm{e}^{-u-x^{2} t / 2 c^{2} u w}}{\sqrt{2 \pi w} c} \mathrm{~d} u \\
= & \frac{1}{\Gamma(\alpha) B\left(d / 2, v_{m}-d / 2+1\right)} \\
& \times \int_{0}^{1} \mathrm{~d} w w^{d / 2-1}(1-w)^{v_{m}-d / 2} \frac{\sqrt{2 t}}{\sqrt{\pi w} c}\left(\frac{\sqrt{t}|x|}{c \sqrt{2 w}}\right)^{\alpha-1 / 2} K_{-\alpha+1 / 2}\left(\frac{\sqrt{2 t}|x|}{c \sqrt{w}}\right),
\end{aligned}
$$

where we have used the change of variable $u=t z$.

Remark 3.7. For $\alpha=1$, we have

$$
\begin{aligned}
& \mathrm{P}\left\{X_{m}\left(B_{d}\left(G_{1}(t)\right)\right) \in \mathrm{d} x \mid N\left(B_{d}\left(G_{1}(t)\right)\right)=n\right\} \\
& \quad=\frac{\mathrm{d} x}{B\left(d / 2, v_{m}-d / 2+1\right)} \int_{0}^{1} \mathrm{~d} w w^{d / 2-1}(1-w)^{v_{m}-d / 2} \frac{\sqrt{2 t}}{2 c \sqrt{w}} \mathrm{e}^{-\sqrt{2 t}|x| / c \sqrt{w}}
\end{aligned}
$$

with $n \geq 1$, which has the probability structure of a Laplace random variable.

Remark 3.8. Let $X$ and $Y$ be two independent random variables distributed as a Gaussian with mean 0 and variance respectively equal to $\sigma^{2}$ and 1 . It is well known that $V=X Y$ has the following density law:

$$
f_{V}(v)=\int_{-\infty}^{+\infty} \frac{\mathrm{e}^{-x^{2} / 2 \sigma^{2}}}{\sqrt{2 \pi} \sigma} \frac{\mathrm{e}^{-v^{2} / 2 x^{2}}}{\sqrt{2 \pi}} \mathrm{d} x=\frac{1}{\pi \sigma} K_{0}\left(\frac{|v|}{\sigma}\right) .
$$

These considerations and Theorem 3.6 permit us to state that, for $\alpha=\frac{1}{2}$ and $d=1$,

$$
\begin{aligned}
& \mathrm{P}\left\{X_{m}\left(\left|W\left(G_{1 / 2}(t)\right)\right|\right) \in \mathrm{d} x \mid N\left(\left|W\left(G_{1 / 2}(t)\right)\right|\right)=n\right\} \\
& \quad=\frac{\mathrm{d} x}{B\left(1 / 2, v_{m}+1 / 2\right)} \int_{0}^{1} \mathrm{~d} w w^{-1 / 2}(1-w)^{v_{m}-1 / 2} \frac{\sqrt{2 t}}{\pi c \sqrt{w}} K_{0}\left(\frac{\sqrt{2 t}|x|}{c \sqrt{w}}\right)
\end{aligned}
$$

holds for each $n \geq 1$. In other words, conditionally on the number of Poisson events during the interval $[0, t]$, the law of $X_{m}\left(\left|W\left(G_{1 / 2}(t)\right)\right|\right)$ is equivalent to the distribution of the product of a standard Gaussian and a normal random variable, mutually independent, with mean 0 and variance $c \sqrt{Z} / \sqrt{2 t}$, where $Z \sim B\left(\frac{1}{2}, v_{m}+\frac{1}{2}\right)$.

\section{Some remarks on random motions in higher spaces}

Let $\boldsymbol{X}_{2}(t)=\left(X_{1}(t), X_{2}(t)\right), t>0$, and $X_{4}(t)=\left(X_{1}(t), X_{2}(t), X_{3}(t), X_{4}(t)\right), t>0$, respectively denote planar and four-dimensional random flights. Then the results presented in the Section 3 can be extended to these random processes.

We also use the following notation: $\boldsymbol{\alpha}_{2}=\left(\alpha_{1}, \alpha_{2}\right), \boldsymbol{\alpha}_{4}=\left(\alpha_{1}, \alpha_{2}, \alpha_{3}, \alpha_{4}\right), \boldsymbol{x}_{2}=\left(x_{1}, x_{2}\right)$, and $\boldsymbol{x}_{4}=\left(x_{1}, x_{2}, x_{3}, x_{4}\right)$. Furthermore, let $\|\cdot\|$ be the euclidean norm and $\langle\cdot, \cdot\rangle$ be the scalar product. As proved in Orsingher and De Gregorio (2007), the characteristic function and the conditional probabilities of the planar random flights are given by

$$
\begin{gathered}
\mathrm{E}\left\{\mathrm{e}^{\mathrm{i}\left\langle\boldsymbol{\alpha}_{2}, \boldsymbol{X}_{2}(t)\right\rangle} \mid N(t)=n\right\}=\frac{\Gamma(n / 2+1) 2^{n / 2}}{\left(c t\left\|\boldsymbol{\alpha}_{2}\right\|\right)^{n / 2}} J_{n / 2}\left(c t\left\|\boldsymbol{\alpha}_{2}\right\|\right), \\
p_{n}\left(\left\|\boldsymbol{x}_{2}\right\|, t\right)=\frac{n}{2 \pi(c t)^{n}}\left(c^{2} t^{2}-\left\|\boldsymbol{x}_{2}\right\|^{2}\right)^{n / 2-1},
\end{gathered}
$$


while, for $\boldsymbol{X}_{4}(t), t>0$, we have

$$
\begin{gathered}
\mathrm{E}\left\{\mathrm{e}^{\mathrm{i}\left\langle\boldsymbol{\alpha}_{4}, X_{4}(t)\right\rangle} \mid N(t)=n\right\}=\frac{\Gamma(n+2) 2^{n+1}}{\left(c t\left\|\boldsymbol{\alpha}_{4}\right\|\right)^{n+1}} J_{n+1}\left(c t\left\|\boldsymbol{\alpha}_{4}\right\|\right), \\
p_{n}\left(\left\|\boldsymbol{x}_{4}\right\|, t\right)=\frac{n(n+1)}{\pi^{2}(c t)^{2 n+2}}\left(c^{2} t^{2}-\left\|\boldsymbol{x}_{4}\right\|^{2}\right)^{n-1} .
\end{gathered}
$$

Therefore, for the planar random flights at a Bessel random time, namely $\left(X_{1}\left(B_{d}(t)\right)\right.$, $\left.X_{2}\left(B_{d}(t)\right)\right), t>0$, by following the same steps as in the proof of Theorem 3.2, for $n \geq 1$, we have

$$
\begin{aligned}
& \mathrm{P}\left\{X_{1}\left(B_{d}(t)\right) \in \mathrm{d} x_{1}, X_{2}\left(B_{d}(t)\right) \in \mathrm{d} x_{2} \mid N\left(B_{d}(t)\right)=n\right\} \\
& \quad=\frac{\mathrm{d} x_{1} \mathrm{~d} x_{2}}{B(d / 2, n / 2-d / 2+1)} \int_{0}^{1} w^{d / 2-1}(1-w)^{n / 2-d / 2} \frac{\mathrm{e}^{-\left\|x_{2}\right\|^{2} / 2 c^{2} t w}}{2 \pi t w c^{2}} \mathrm{~d} w
\end{aligned}
$$

with $n / 2>d / 2-1$, while, for $\left(X_{1}\left(G_{\alpha}(t)\right), X_{2}\left(G_{\alpha}(t)\right)\right), t>0$, we have

$$
\begin{aligned}
\mathrm{P}\left\{X_{1}\left(G_{\alpha}(t)\right) \in \mathrm{d} x_{1}, X_{2}\left(G_{\alpha}(t)\right) \in \mathrm{d} x_{2} \mid N\left(G_{\alpha}(t)\right)=n\right\} \\
=\frac{\mathrm{d} x_{1} \mathrm{~d} x_{2}}{\Gamma((\alpha+1) / 2) B(\alpha / 2, n / 2-\alpha / 2+1)} \\
\quad \times \int_{0}^{1} \mathrm{~d} w w^{\alpha / 2-1}(1-w)^{n / 2-\alpha / 2} \frac{t}{\sqrt{\pi w} c}\left(\frac{t\left\|x_{2}\right\|}{2 c \sqrt{w}}\right)^{\alpha / 2} K_{-\alpha / 2}\left(\frac{t\left\|x_{2}\right\|}{c \sqrt{w}}\right),
\end{aligned}
$$

which hold if $n / 2>\alpha / 2-1$. We observe that from probability (4.1) for $d=1$ we again obtain result (3.3) of Beghin and Orsingher (2009). Similar considerations hold for the fourdimensional random flights.

The random flights in higher spaces have directions uniformly distributed on a multidimensional hypersphere. It would be interesting to consider a model, for example in the plane, with a different density law with respect to the uniform law. Let us consider a planar random flight with density law similar to (2.1), for example,

$$
f(\theta)=\frac{1}{\pi} \sin ^{2} \theta, \quad \theta \in[0,2 \pi] .
$$

Therefore, we obtain a random motion with drift, which is persistent along a specific portion of the plane. Hence, in order to calculate the characteristic function, we need to evaluate $\int_{0}^{2 \pi} \exp \{\mathrm{i} z(\alpha \cos \theta+\beta \sin \theta)\} \sin ^{2} \theta \mathrm{d} \theta$. We evaluate this integral as follows:

$$
\begin{aligned}
\int_{0}^{2 \pi} & \exp \{\mathrm{i} z(\alpha \cos \theta+\beta \sin \theta)\} \sin ^{2} \theta \mathrm{d} \theta \\
= & \sum_{k=0}^{\infty} \frac{(\mathrm{i} z)^{k}}{k !} \int_{0}^{2 \pi}(\alpha \cos \theta+\beta \sin \theta)^{k} \sin ^{2} \theta \mathrm{d} \theta \\
= & \sum_{k=0}^{\infty} \frac{(\mathrm{i} z)^{k}}{k !} \sum_{r=0}^{k}\left(\begin{array}{l}
k \\
r
\end{array}\right) \alpha^{r} \beta^{k-r} \int_{0}^{2 \pi} \cos ^{r} \theta \sin ^{k-r+2} \theta \mathrm{d} \theta
\end{aligned}
$$

Now, the last integral has to be split into two parts, i.e. $\int_{0}^{2 \pi}=\int_{0}^{\pi}+\int_{\pi}^{2 \pi}$. Hence, by performing the change of variable $\theta^{\prime}=\theta-\pi$ in the second integral, we observe that the previous sum is equal to 0 if $k$ is odd. By splitting the first integral on $(0, \pi / 2)$ and using a change of variable analogous to the previous change, we find that the sum is equal to 0 if $r$ is odd. Therefore, we 
can write

$$
\begin{aligned}
& \int_{0}^{2 \pi} \exp \{\mathrm{iz}(\alpha \cos \theta+\beta \sin \theta)\} \sin ^{2} \theta \mathrm{d} \theta \\
& =4 \sum_{k=0}^{\infty} \frac{(\mathrm{i} z)^{2 k}}{(2 k) !} \sum_{r=0}^{k}\left(\begin{array}{l}
2 k \\
2 r
\end{array}\right) \alpha^{2 r} \beta^{2(k-r)} \int_{0}^{\pi / 2} \cos ^{2 r} \theta \sin ^{2(k-r+1)} \theta \mathrm{d} \theta \\
& =2 \sum_{k=0}^{\infty}(-1)^{k} \frac{z^{2 k}}{(2 k) !} \sum_{r=0}^{k}\left(\begin{array}{l}
2 k \\
2 r
\end{array}\right) \alpha^{2 r} \beta^{2(k-r)} \frac{\Gamma(r+1 / 2) \Gamma(k-r+1+1 / 2)}{\Gamma(k+2)} \\
& =2 \pi \sum_{k=0}^{\infty}(-1)^{k} \frac{z^{2 k}}{(2 k) !} \sum_{r=0}^{k}\left(\begin{array}{l}
2 k \\
2 r
\end{array}\right) \alpha^{2 r} \beta^{2(k-r)} \frac{2^{-2 k} \Gamma(2 r) \Gamma(2(k-r+1))}{\Gamma(k+2) \Gamma(r) \Gamma(k-r+1)} \\
& =2 \pi \sum_{k=0}^{\infty}(-1)^{k} \frac{z^{2 k}}{\Gamma(k+2) 2^{2 k}} \sum_{r=0}^{k} \frac{2(k-r)+1}{2 r \Gamma(r) \Gamma(k-r+1)} \alpha^{2 r} \beta^{2(k-r)} \\
& =2 \pi \sum_{k=0}^{\infty}(-1)^{k} \frac{z^{2 k}}{(k+1) ! 2^{2 k}}\left\{\sum_{r=0}^{k} \frac{\alpha^{2 r} \beta^{2(k-r)}}{2(r !)(k-r) !}+\sum_{r=0}^{k} \frac{\alpha^{2 r} \beta^{2(k-r)}}{r !(k-r-1) !}\right\} \\
& =2 \pi \sum_{k=0}^{\infty}(-1)^{k} \frac{z^{2 k}}{(k+1) ! 2^{2 k}}\left\{\frac{1}{2(k !)} \sum_{r=0}^{k}\left(\begin{array}{l}
k \\
r
\end{array}\right) \alpha^{2 r} \beta^{2(k-r)}\right. \\
& \left.+\frac{\beta^{2}}{(k-1) !} \sum_{r=0}^{k-1}\left(\begin{array}{c}
k-1 \\
r
\end{array}\right) \alpha^{2 r} \beta^{2(k-1-r)}\right\} \\
& =2 \pi\left\{\sum_{k=0}^{\infty} \frac{(-1)^{k} z^{2 k}}{2(k+1) ! k ! 2^{2 k}}\left(\sqrt{\alpha^{2}+\beta^{2}}\right)^{2 k}\right. \\
& \left.+\beta^{2} \sum_{k=1}^{\infty} \frac{(-1)^{k} z^{2 k}}{(k+1) !(k-1) ! 2^{2 k}}\left(\sqrt{\alpha^{2}+\beta^{2}}\right)^{2(k-1)}\right\} \\
& =2 \pi\left\{\frac{J_{1}\left(z \sqrt{\alpha^{2}+\beta^{2}}\right)}{z \sqrt{\alpha^{2}+\beta^{2}}}-\beta^{2} \sum_{l=0}^{\infty} \frac{(-1)^{l}}{l !(l+2) !}\left(\frac{z}{2}\right)^{2 l+2}\left(\sqrt{\alpha^{2}+\beta^{2}}\right)^{2 l}\right\} \\
& =2 \pi\left\{\frac{J_{1}\left(z \sqrt{\alpha^{2}+\beta^{2}}\right)}{z \sqrt{\alpha^{2}+\beta^{2}}}-\frac{\beta^{2}}{\alpha^{2}+\beta^{2}} J_{2}\left(z \sqrt{\alpha^{2}+\beta^{2}}\right)\right\} .
\end{aligned}
$$

Then, in view of the proof of Theorem 2.1, the characteristic function becomes

$$
\begin{aligned}
& \mathrm{E}\left\{\mathrm{e}^{\mathrm{i}\left\langle\boldsymbol{\alpha}_{2}, \boldsymbol{X}_{2}(t)\right\rangle} \mid N(t)=n\right\} \\
& =\frac{n !}{t^{n}} 2^{n+1} \int_{0}^{t} \mathrm{~d} s_{1} \cdots \\
& \times \int_{s_{n-1}}^{t} \mathrm{~d} s_{n} \prod_{j=1}^{n+1}\left\{\frac{J_{1}\left(c\left(s_{j}-s_{j-1}\right)\left\|\boldsymbol{\alpha}_{2}\right\|\right)}{c\left(s_{j}-s_{j-1}\right)\left\|\boldsymbol{\alpha}_{2}\right\|}-\frac{\alpha_{2}^{2}}{\left\|\boldsymbol{\alpha}_{2}\right\|^{2}} J_{2}\left(c\left(s_{j}-s_{j-1}\right)\left\|\boldsymbol{\alpha}_{2}\right\|\right)\right\} .
\end{aligned}
$$

From (4.2), it emerges that, as expected, an asymmetry is introduced by $f(\theta)$, because the particle will tend to maintain the same direction. Moreover, the inversion of the characteristic function is quite difficult; therefore, it does not seem to be possible to obtain the explicit probability distribution of $\boldsymbol{X}_{2}(t)$ at time $t$ by means of this approach. 


\section{Acknowledgement}

The author wishes to thank the anonymous referee for carefully reading an earlier version of the manuscript.

\section{References}

AlloubA, H. (2002). Brownian-time processes: the PDE connection. II. And the corresponding Feynman-Kac formula. Trans. Amer. Math. Soc. 354, 4627-4637.

Beghin, L. AND Orsingher, E. (2009). Fractional Poisson processes and related planar random motions. Electron. J. Prob. 14, 1790-1827.

Burdzy, K. (1993). Some path properties of iterated Brownian motion. In Seminar on Stochastic Processes, eds E. Cinlar, K. L. Chung and M. J. Sharpe, Birkhäuser, Boston, MA, pp. 67-87.

Burdzy, K. And Khoshnevisan, D. (1998). Brownian motion in a Brownian crack. Ann. Appl. Prob. 8, $708-748$.

DeBlassie, R. D. (2004). Iterated Brownian motion in an open set. Ann. Appl. Prob. 14, 1529-1558.

De Gregorio, A. and Iacus, S. M. (2008). Parametric estimation for standard and geometric telegraph process observed at discrete times. Statist. Infer. Stoch. Process. 11, 249-263.

De Gregorio, A. And Orsingher, E. (2006). Some results on random flights. Sci. Math. Jpn. 64, 351-356.

Di Crescenzo, A. (2001). On random motions with velocities alternating at Erlang-distributed random times. Adv. Appl. Prob. 33, 690-701.

Di Crescenzo, A. And Pellerey, F. (2002). On prices' evolutions based on geometric telegrapher's process. Appl. Stoch. Models Business Industry 18, 171-184.

Foong, S. K. And Kanno, S. (1994). Properties of the telegrapher's random process with or without a trap. Stoch. Process. Appl. 53, 147-173.

Gradshteyn, I. S. AND RyzhiK, I. M. (1980). Table of Integrals, Series and Products. Academic Press, New York.

Hillen, T. And Stevens, A. (2000). Hyperbolic models for chemotaxis in 1-D. Nonlinear Anal. Real World Appl. 1, 409-433.

Holmes, E. E., Lewis, M. A., Banks, J. E. And Veit, R. R. (1994). Partial differential equations in ecology: spatial interactions and population dynamics. Ecology 75, 17-29.

IACUs, S. M. AND YoshidA, N. (2009). Estimation for discretely observed telegraph process. Theory Prob. Math. Statist. 78, 37-47.

Kolesnik, A. D. (2006). A four-dimensional random motion at finite speed. J. Appl. Prob. 43, 1107-1118.

Kolesnik, A. D. AND Orsingher, E. (2005). A planar random motion with an infinite number of directions controlled by the damped wave equation. J. Appl. Prob. 42, 1168-1182.

Khoshnevisan, D. And Lewis, T. M. (1996). The uniform modulus of continuity of iterated Brownian motion. J. Theoret. Prob. 9, 317-333.

MaZzA, C. AND Rullière, D. (2004). A link between wave governed random motions and ruin processes. Insurance Math. Econom. 35, 205-222.

NANE, E. (2006). Iterated Brownian motion in bounded domains in $\mathbb{R}^{n}$. Stoch. Process. Appl. 116, 905-916.

OrSINGHER, E. (1990). Probability law, flow function, maximum distribution of wave-governed random motions and their connections with Kirchoff's laws. Stoch. Process. Appl. 34, 49-66.

Orsingher, E. AND BEGHIN, L. (2009). Fractional diffusion equations and processes with randomly varying time. Ann. Prob. 37, 206-249.

Orsingher, E. And De Gregorio, A. (2007). Random flights in higher spaces. J. Theoret. Prob. 20, $769-806$.

Ratanov, N. (2007a). A jump telegraph model for option pricing. Quant. Finance 7, 575-583.

Ratanov, N. (2007b). Jump telegraph processes and financial markets with memory. J. Appl. Math. Stoch. Anal. 2007, $72326,19 \mathrm{pp}$.

Stadje, W. (1987). The exact probability distribution of a two-dimensional random walk. J. Statist. Phys. 46, 207-216.

Stadje, W. (1989). Exact probability distributions for noncorrelated random walk models. J. Statist. Phys. 56, $415-435$

Stadje, W. And Zacks, S. (2004). Telegraph processes with random velocities. J. Appl. Prob. 41, 665-678.

ZACKS, S. (2004). Generalized integrated telegraph processes and the distribution of related stopping times. J. Appl. Prob. 41, 497-507.

WeIss, G. H. (2002). Some applications of persistent random walks and the telegrapher's equation. Physica A 311, $381-410$ 